\title{
Novel measures of Morris water maze performance that use vector field maps to assess accuracy, uncertainty, and intention of navigational searches
}

\author{
Prabod Kumar Meenakshi $^{1}$ () | Dhruv Mehrotra ${ }^{1,2}$ ( ) | N. Nruthyathi ${ }^{1,2}$ | \\ Daniel Almeida-Filho $^{3}$ | Yong-Seok Lee ${ }^{4}$ | Alcino Silva ${ }^{3}$ | J. Balaji ${ }^{1}$ (c)
}

${ }^{1}$ Centre for Neuroscience, Indian Institute of Science, Bengaluru, India

${ }^{2}$ Undergraduate Program of Indian Institute of Science, Bengaluru, India

${ }^{3}$ Departments of Neurobiology, Psychology, Psychiatry, Integrative Center for Learning and Memory and Brain Research Institute, University of California Los Angeles, Los Angeles, California, USA

${ }^{4}$ Departments of Physiology, Biomedical Sciences, Neuroscience Research Institute, Seoul National University College of Medicine, Seoul, South Korea

\section{Correspondence}

Balaji Jayaprakash, Centre for Neuroscience, Indian Institute of Science, Bengaluru, 560012, India.

Email: jbalaji@iisc.ac.in

\section{Funding information}

Council for Scientific and Industrial Research, Human Resource Development Group (CSIR HRDG), India, Grant/Award Number: CSIR09/079(2697)/2016-EMR-I; Department of Biotechnology, Ministry of Science and Technology, DBT IISc Partnership; Department of Science and Technology, Ministry of Science and Technology, India, Ramanujan Fellowship; National Institutes of Health, Grant/Award Number: R01 MH084315; Pratiksha Trust; Science and Engineering Research Board, Grant/Award Number: EMR/2017/004155

\begin{abstract}
Most commonly used behavioral measures for testing learning and memory in the Morris water maze (MWM) involve comparisons of an animal's residence time in different quadrants of the pool. Such measures are limited in their ability to test different aspects of the animal's performance. Here, we describe novel measures of performance in the MWM that use vector fields to capture the motion of mice as well as their search pattern in the maze. Using these vector fields, we develop quantitative measures of performance that are intuitive and more sensitive than classical measures. First, we describe search patterns in terms of vector field properties and use these properties to define three metrics of spatial memory namely Spatial Accuracy, Uncertainty and, Intensity of Search. We demonstrate the usefulness of these measures using four different data sets including comparisons between different strains of mice, an analysis of two mouse models of Noonan syndrome (NS; Ptpn11 D61G and Ptpn11 N308D/+), and a study of goal reversal training. Importantly, besides highlighting novel aspects of performance in this widely used spatial task, our measures were able to uncover previously undetected differences, including in an animal model of NS, which we rescued with the mitogen activated protein kinase kinase (MEK) inhibitor SL327. Thus, our results show that our approach breaks down performance in the MWM into sensitive measurable independent components that highlight differences in spatial learning and memory in the MWM that were undetected by conventional measures.
\end{abstract}

\section{KEYWORDS}

convergence hotspots, intensity of search, Morris water maze, search center accuracy, search center spread, vector field properties

\section{1 | INTRODUCTION}

The Morris water maze (MWM) task and its modifications have been extensively used in the field of neuroscience to study a variety of cognitive phenomena, especially related to hippocampal-

Abbreviations: ME, maximum entropy; MWM, Morris water maze; ROI, region of interest. dependent learning and memory (Brandeis et al., 1989; D'Hooge \& De Deyn, 2001; Redish \& Touretzky, 1998). In the original or reference version of the MWM task, rodents, over multiple trials, learn the spatial location of a hidden platform submerged in a pool of water using distal cues (R. Morris, 1984; R. G. M. Morris, 1981). Learning and memory is assessed with multiple performance measures, such as time to the platform during training, and proximity 
during probe trials given at different times during training. During a probe trial, the platform is removed, and an animal is allowed to search for the platform usually for 60s. Search behavior during a probe trial is typically the most sensitive measure of an animal's spatial learning and memory. Traditionally, learning and memory is usually assessed by comparing the time the animal spent in the quadrant of the pool where the platform was located during training (target quadrant) with the time spent in the other three quadrants (Petrosini et al., 1998). Time spent in zones or areas of multiple sizes centered in the platform's location is also used as a parameter for measuring spatial learning and memory as this provides a more detailed readout for the spatial distribution of the animal's searches.

Additional methods to measure the animal's performance have been proposed over the years (Dalm et al., 2000; Pereira \& Burwell, 2015; Wolfer \& Lipp, 1992), including a platform proximity measure (Gallagher et al., 1993). This approach calculates the average distance of the rodent from the center of the platform location, and it is effective in differentiating between search strategies of equal path length (i.e., a focused search near the platform and a diffused search around the platform). Incidentally, this proximity measure has been shown to be more sensitive than classical measures described above, including quadrant search measures, zonal, and platform crossings (Maei, Zaslavsky, Teixeira, \& Frankland, 2009).

Another approach to quantify performance in the MWM uses more complex methods that classify the animal's swim trajectories into different path strategies. These approaches capture qualitative measures of performance in the water maze task (Cooke et al., 2019; Garthe et al., 2009; Gehring et al., 2015; Graziano et al., 2003; Vouros et al., 2018). A parameter free machine learning based algorithm was also developed to classify MWM search strategies (Overall et al., 2020). This approach is automated and uses trajectories to assign performances in individual trials to different classes. Thus, they are useful to develop descriptive accounts of an animal's performance with defined search categories.

A measure was also proposed that uses the concept of entropy from information theory (Maei, Zaslavsky, Wang, et al., 2009). This entropy-based measure (measure $H$ ) describes the extent of search at the platform location as well as the focus of this search (Maei, Zaslavsky, Wang, et al., 2009). The rational for this approach is that rodents improve the focus or "organize" their search efforts to locate the platform as a function of learning. Thus, with training swim trajectories go from a highly disorganized to a more organized state. Computing the entropy of the swim trajectories (summation of entropy in path variance $\left[H_{\text {path }}\right]$ and entropy in error variance $\left[H_{\text {error }}\right]$ provides a more sensitive measure than the simpler proximity measure. However, the improved sensitivity of the $H$ measure is dependent on the relative weighting of its components $\left(H_{\text {path }}\right.$ and $\left.H_{\text {error }}\right)$ in a given experimental setting, since one individual component maybe be more sensitive to specific search strategies. Such nonlinear estimates, despite being highly sensitive, do not provide a uniform scale of measurement that is intuitive and easy to use. Additionally, the $H$ measure primarily estimates the disorderliness of performances in the MWM and does not reflect specific spatial or navigational properties of these performances.

Many of the above methods focus on analyzing the trajectories in a navigation task and rely on measuring differences in path accuracy. Path accuracy differences in turn are then used as an indicator for the differences in spatial memory. Navigation requires the animal represent the space and its subsequent use to find the optimal/efficient trajectories to reach different locations in space. While path inaccuracies or errors are good to follow and measure the differences in navigational ability they do not always correspond to memory (Eichenbaum, 2017; H. Eichenbaum \& Cohen, 2014).

Here, we propose new measures based on vector fields constructed from velocity components that are oriented toward a specific point in the water maze. Our measures stem from the observation that animals tend to slow down at and around the platform location during probe trials with the expectation of landing on the platform. This behavior gains prominence as animals learns the location of the platform. Such slowing down also occurs when they are approaching the wall of the pool. In addition, animals show similar slowing down when they overshoot the platform position and turn around. Thus, the measures described here to understand the properties of these vectors and their spatial distribution as a vector field could provide valuable information regarding how the animal perceives the location of the platform. We proposed that the framework and associated measures introduced here to measure the location in the pool about which the slowing down occurs provide a much-improved strategy to access their spatial memory retention in the MWM.

The measures introduced here enabled us to identify subtle memory deficits in the MWM. We propose that vector maps of the velocity vector component along the occupancy center $\left(V_{\| t}\right)$ and its orthogonal component $\left(V_{\perp t}\right)$, contain information that reflects the intention of the animal's movement. Specifically, we propose that the velocity vector component along the occupancy center $\left(V_{\| t}\right)$ measures the animal's movement that contributes to its approach to the platform location. In addition, the velocity vector component orthogonal to the occupancy center $\left(V_{\perp t}\right)$ measures the animal's movement that contributes to motion around the occupancy center. We also calculate the vector field properties, namely divergence on $V_{\| t}$, to describe the rate of change of these measures in 2D space. Specifically, we propose that spatially localized negative divergence peaks of $V_{\| t}$ reveal convergence hotspots or putative platform search centers $\left(P_{c s}\right)$. Using the search center measure, we quantify spatial memory in terms of three independent metrics, namely accuracy, uncertainty, and intensity of search.

We argue that unlike the previously proposed measures, the three metrics introduced here, based on divergence field properties, capture the nature of spatial memory independently of factors 
unrelated to learning and memory-based phenomena, such as motor performance.

\section{2 | THEORY}

We have used velocity-based vector fields to develop an approach that captures key components of an animals spatial learning and memory performance in the MWM. This approach depends on three independent measures, namely accuracy, uncertainty, and intensity of search. A detailed and a formal description of the method is presented in the Appendix A. For easy of reading and to provide context to subsequent results we briefly describe the approach here. We start by identifying a place in the pool where the animal is most likely to be found given its trajectory and term it "occupancy center" $\left(P_{o c}\right)$. Following this we measure the contribution of the animal's movement toward the occupancy center. Vector projections that reflect the animal's intention to move toward the occupancy center from different points in the pool are constructed. Next, we measure if such "intention" vector fields converge in space, and we identify such peaks of convergence as putative search centers. Having identified the putative search centers, we calculate the accuracy of spatial memory as the distance of putative search center from the platform center. We note that this measure is independent of the path taken by the animal and only depends on how the velocity changes are distributed about a point in space. Additionally, we also define uncertainty to capture how widespread the search for the platform is and intensity to capture the intention of search about the putative search center. Throughout the text we use these three measures to characterize and follow behavioral changes of mice in MWM. In brief these are defined as

$$
\operatorname{Accuracy}\left(\alpha_{c s}\right)=\left(1-\frac{d_{c s}}{e}\right) \times 100
$$

where $d_{c s}$ is the distance of the search center from platform; $e$ is the maximum error (distance of the platform from the farthest boundary).

$$
\text { Uncertainity }\left(d_{R S}\right)=\frac{\sigma_{c S}}{d_{P_{L}}}
$$

where $\sigma_{c s}$ is the FWHM of the peak; $d_{P_{L}}$ is the diameter of the platform.Additionally, we define absolute and relative intensity of search as follows:

Absolute intensity $\left(a l_{c s}\right)=-I_{\text {div }}\left(x_{0}, y_{0}\right)$,

where $I_{\text {div }}$ is the divergence at peak.

$$
\text { Relative intensity }\left(r I_{c S}\right)=\frac{a l_{c s}}{I_{\max }}
$$

where $I_{\max }$ is the maximal divergence in the pool.

\section{3 | RESULTS}

\subsection{Swim trajectories described as velocity vector fields reveal that the speed of intentional movement varies over the pool space as a function of training}

In an effort to assess spatial learning and memory, we first sought to describe the movement of the mice using the most basic kinematic measure, that is, velocity. For this we used a previously published water maze data set from a mouse model of Noonan syndrome (NS; Romano et al., 2010; Tartaglia \& Gelb, 2005), the Ptpn11 ${ }^{\text {D61G/+ }}$ mice and their littermate controls (Lee et al., 2014). Using a time series of position data obtained from swim trajectories, we constructed velocity vectors (Equation (A3), Figure 1c). These vectors describe the distance covered per unit time as the mice searched for the platform (Figure 2a-d). When we measured the magnitude of the velocity vector (speed), as a function of the radial distance from the platform, we noted that it was invariant across space when the mice had no knowledge of the platform's location (Figure 3a). However, we observed that after training, there was a reduction in the speed of the mice closer to the platform location during the probe trials (Red Arrows in Figure $3 b-d$ ). There was also a reduction in speed of the mice when they were closer to the wall of the pool. Further, the reduced speeds around platform position correlates with proximity measure (Figure $\mathrm{S} 8 \mathrm{~d}$ ).

We reasoned that studying the variation of speed across the spatial dimension would reveal the regions where the mice slowed down in their effort to locate the platform. Specifically, we propose that the divergence on the velocity vector field reveals the "sources" and "sinks" in that field, that is, the regions where the mice swim away from with high speed (divergence points), and regions where the mice swim into with low speed (convergence points). In general, we recognize that at any given time, only a fraction of an animal's movement, and not necessarily the entire movement, may be oriented toward its intended direction. Given this, we generated a vector field around the point in the pool where the mice are most likely to be found, that is, the occupancy center. We propose that the regions of low residence typically result from incidental occupancy of these locations merely by being on the navigational path, rather than being the locations where the mice intent or decide to go. We identified the high occupancy regions as the regions where the mice intended to stay. We used maximum entropy (ME) to demarcate these regions as the regions where the mice are more likely to sample. We calculated the center of mass of these most likely occupied regions to get the occupancy center as shown in Figure 2e-h. We carried out this analysis for wild type (WT) littermates of Ptpn11 ${ }^{\mathrm{D} 61 \mathrm{G} /+}$ mice on training day 1 , as well as during probe trials given at the end of days 3, 5, and 7 of training. With this occupancy center, we described the swim trajectories of the mice in terms of a velocity vector component along the occupancy center (Equation (A6)).

We reasoned that if a part, and not necessarily the whole of the movement, reflects the mice's intentions, then we might be able to see a change as a function of training. Accordingly, we observed that the mice show movements that are more aligned to the occupancy 


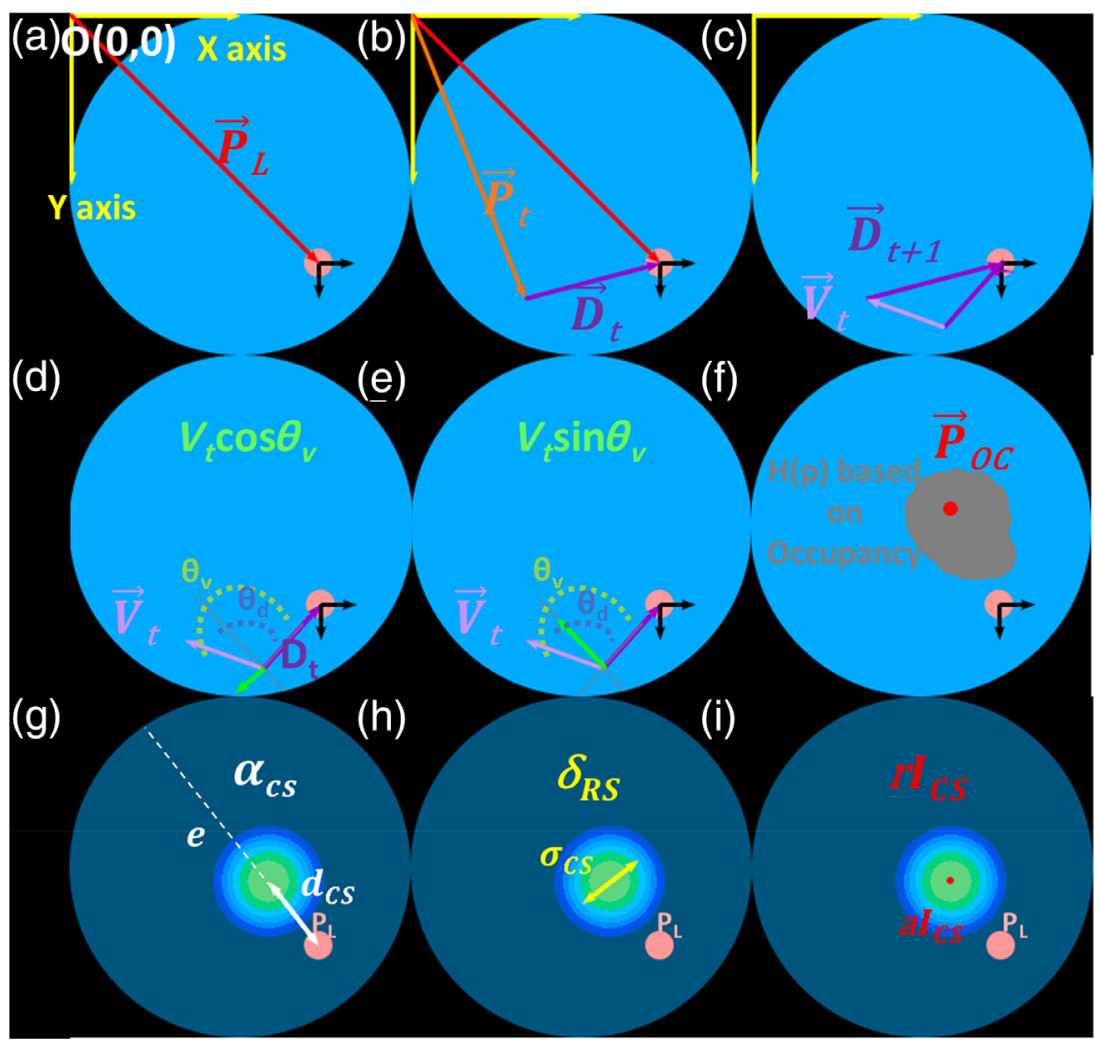

FIGURE 1 Schematic representation of the coordinate system used to describe the water maze pool and mouse trajectory as a velocity vector field. Three metrics derived from the velocity vector field that we define, and use, measure the quality of the spatial memory, namely accuracy, uncertainty, and intensity of search (see Section 2 for details). (a) The co-ordinate system and the reference frame that is used to represent the video frame data point acquired from a trial/session of a navigational behavior. Origin of the coordinate system $(O[0,0])$ is located at the top left corner of the image. $P_{L}$ (red arrow) is position vector of the platform (pink circle) center. (b) We define a position vector $P_{t}$ (orange vector) as the point at which the mouse is located at a given time/video frame ( $t$ ). Thus, the current position of the mouse to the platform location is given by the displacement vector $D_{t}$ (purple vector). (c) Velocity vector $V_{t}$ (light purple vector) is calculated as the difference in displacement vectors obtained from consecutive video frames. The velocity vector describes the movement of the mouse. It is used as the base measure for developing three metrics for assessing spatial memory and its retention. (d) Resolving velocity vector into its components, a component along a vector pointing toward the platform from current position $\left(V_{\| P_{L}}=V_{t} \cos \theta_{v}\right.$, green vector) measures the mouse's movement that contributes toward or away from the platform. In this schematic the component is pointing away from the platform. (e) Similarly, the velocity vector component orthogonal to the above component could represent the movement contributing to circular motion of the mice centered about the platform $\left(V_{\perp P_{L}}=V_{t} \sin \theta_{v}\right.$, green vector). (f) However, to assess the quality of spatial memory, we resolve the velocity vector into its components with respect to the occupancy center $\left(\vec{P}_{\text {oc }}\right)$ given by the center of mass (COM) of occupancy. COM calculated on a maximum entropy thresholded occupancy image and represents the most likely region occupied by the mouse during probe trial. We create a vector field from these components to describe the mouse's intentional movement toward the occupancy center as well as the extent of circling about that point.

Analyzing the field properties, namely divergence and curl, allows us to assess the spatial memory. The divergence heat map reveals convergence hotspots, the peak of which represents the putative search centers $\left(\vec{P}_{c s}\right)$. (g) We define accuracy in spatial memory $\left(\alpha_{c s}\right)$ as a measure that reflects the accuracy with which the mouse remembers the platform location. It is expressed as percentage of 1 minus the fractional error, where the error $\left(d_{c s}\right)$ is the displacement between the search center $\vec{P}_{c s}$ and the platform location $\mathrm{P}_{\mathrm{L}}$, and the maximum possible error (e, white dashed line) is the displacement of the farthest boundary/periphery of the pool from the platform. (h) We define our second metric, uncertainty in search, as the spatial spread of the search $\left(\sigma_{c s}\right)$, defined as the full width half maxima (FWHM) in $x$ axis and $y$ axis of the convergence peak. We describe the uncertainty in terms of relative search diameter $\left(\delta_{R S}\right)$, where the search diameter is normalized to platform diameter $\left(d_{P L}\right)$. (i) Lastly, intensity of search $\left(I_{c s}\right)$ reveals the intensity or intent with which the mouse moves to the search center. The absolute intensity of search $\left(a I_{c s}\right)$ is represented by the convergence value at the search center, whereas the relative intensity of search $\left(r l_{c s}\right)$ is the convergence value normalized to the maximum convergence value in the pool space. Since the convergence value looks at the rate of change of the velocity vectors in a small area, the measure is not confounded by differences in swim speed among mice

center during the probe trials (Figures $3 \mathrm{f}-\mathrm{h}$ and $\mathrm{S} 8 \mathrm{f}-\mathrm{h}$ ) compared with that of training (Figures $3 e$ and $\mathrm{S} 8 \mathrm{e}$ ). Thus, we used the velocity vector component, defined in Equation (A6), to construct vector fields and obtain the divergence measure (Equation (A8)). Figure $3 i$ depicts the surface of the divergence measure estimated using the performance of WT mice during training day 1 (D1; left panel) and probe day 7 (PD7; right panel). This analysis reveals a valley near the platform location as the mice's performance improves. 

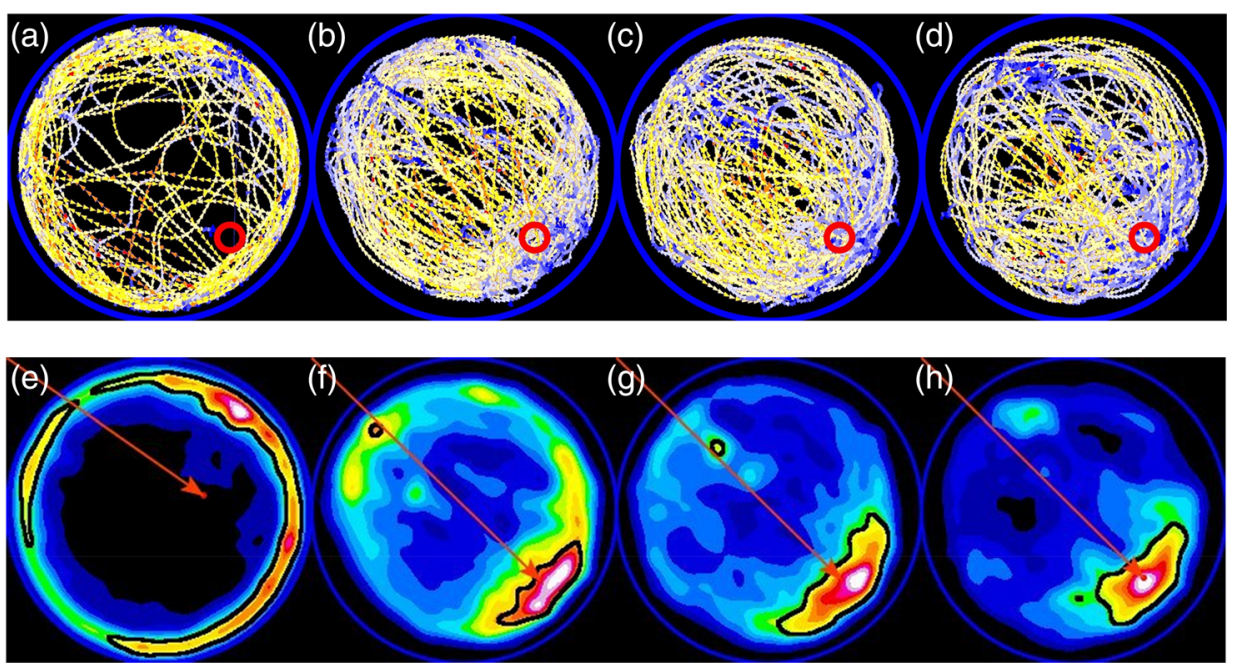

FIGURE 2 Velocity vector field, residence time heat map defines the occupancy center. The occupancy center $\left(\vec{P}_{\text {oc }}\right)$ is shown for the population data of Ptpn11+/+ wild type mice. (a-d) show the velocity vector field on training day 1 (D1), probe day 3 (PD3), probe day 5 (PD5), and probe day 7 (PD7), respectively. LUT scale: 0-8 in pixels/frame. (e-h) show the occupancy heat map on D1, PD3, PD5, and PD7, respectively. The regions enclosed in black outline represent the most likely regions of occupancy obtained using maximum entropy threshold (see Appendix A: Theory for details.) Based on the most likely regions, the occupancy center is calculated (shown as a red point). The position vector of this point in the image reference frame is shown as red arrows. See Figures S1 and S2 for velocity vector field map, residence time heat map showing most likely occupied region, and occupancy centers for all strains/datasets used for analysis in this study. The images are Gaussian smoothed (radius $=6$ ) to approximate the point object to real world dimensions

\subsection{The negative divergence in the vector field of the velocity component along the occupancy center, rather than velocity itself, describes the putative search centers as convergence hotspots for a given swim pattern}

To describe swim patterns during probe trials, we constructed a velocity vector field based on the movement of the mice. Together with the velocity vector field, we also constructed a vector field of the velocity component along the occupancy center (Equation (A6)). We rationalized that the velocity component along the occupancy center quantifies the effect of an intentional movement toward the occupancy center. We also determined convergence peaks on these vector fields. Given our assertion that for a given swimming pattern, a convergence peak represents the point the mice swim toward, the convergence peak is a proxy for the perceived platform location, as it represents a mouse's putative search center. Figure S3 provides the graphical representation of the workflow.

We can generate such a convergence heat map for an individual mouse as well as for a group of mice. For example, we show the velocity vector field maps for an individual Ptpn $11^{+/+}$mouse in Figure $4 \mathrm{a}$, as well as the velocity vector field for a group of Ptpn $11^{+/+}$ mice ( $n=15$; Figure $4 c$ ) for their trajectories on probe day 7 . The divergence map calculated on the vector fields shows the presence of convergence peaks near the platform (Figure $4 \mathrm{f}, \mathrm{h}$ ). In comparison, the divergence map calculated on the velocity component shows a sharp, well-defined convergence peak near the platform site for both the individual and group's trajectories (Figures $3 i$ and $4 \mathrm{j}, \mathrm{l}$ ). While the spatial sampling of the pool during the probe trial by an individual mouse is sufficient to detect convergence peaks (thus allowing us to identify putative search centers for each individual mouse), for subsequent analyses, we used the group trajectories.

\subsection{Accuracy, uncertainty and intensity of search centers are informative in distinguishing differences between spatial memory of two strains of mice: $B A L B / c J$ and SWR/J}

We analyzed the WM swim trajectories of the BALB/cJ and SWR/J mouse strains. The divergence heat maps show a distinct convergence hotspot for both, BALB/cJ $(n=10)$ and SWR/J $(n=9 / 10)$, groups of mice on all three probe days (i.e., PD3, PD7, and PD10 (Figure 5a-d).

On calculating the accuracy in search for BALB/cJ mice, we observed as expected that the accuracy increases progressively and reaches an asymptote with additional training (Figure $5 \mathrm{e}$, Green solid bars, $\alpha_{c s}=80 \% \pm 2.2 \%$ [PD3], $\alpha_{c s}=91 \% \pm 1.3 \%$ [PD7], $\alpha_{c s}=95 \%$ $\pm 1.5 \%$ [PD10]; Table S2), reflecting accurate knowledge of the platform's location. Comparatively, SWR/J mice show an accuracy worse than chance levels (as defined in the theory section Equation (A12)) on a probe trial at day 3 (PD3) and PD7, indicating that the mice are searching away from both the pool center and the platform's location. Further training of SWR/J mice marginally improves the accuracy on PD10 (Figure 5f, Blue solid bars, $\alpha_{c s}=51 \% \pm 0.66 \%$ [PD3], $\alpha_{c s}=61 \% \pm 1.15 \%$ (PD7), $\alpha_{c s}=74 \% \pm 1.41 \%$ [PD10]). The occupancybased quadrant and platform measures show that SWR/J mice do not preferentially reside in the target quadrant (P4/Q4) on PD3, but with training, these mice preferentially reside in the training platform zone on PD10 (Figure S4h,j). However, our analysis of swim trajectories shows 

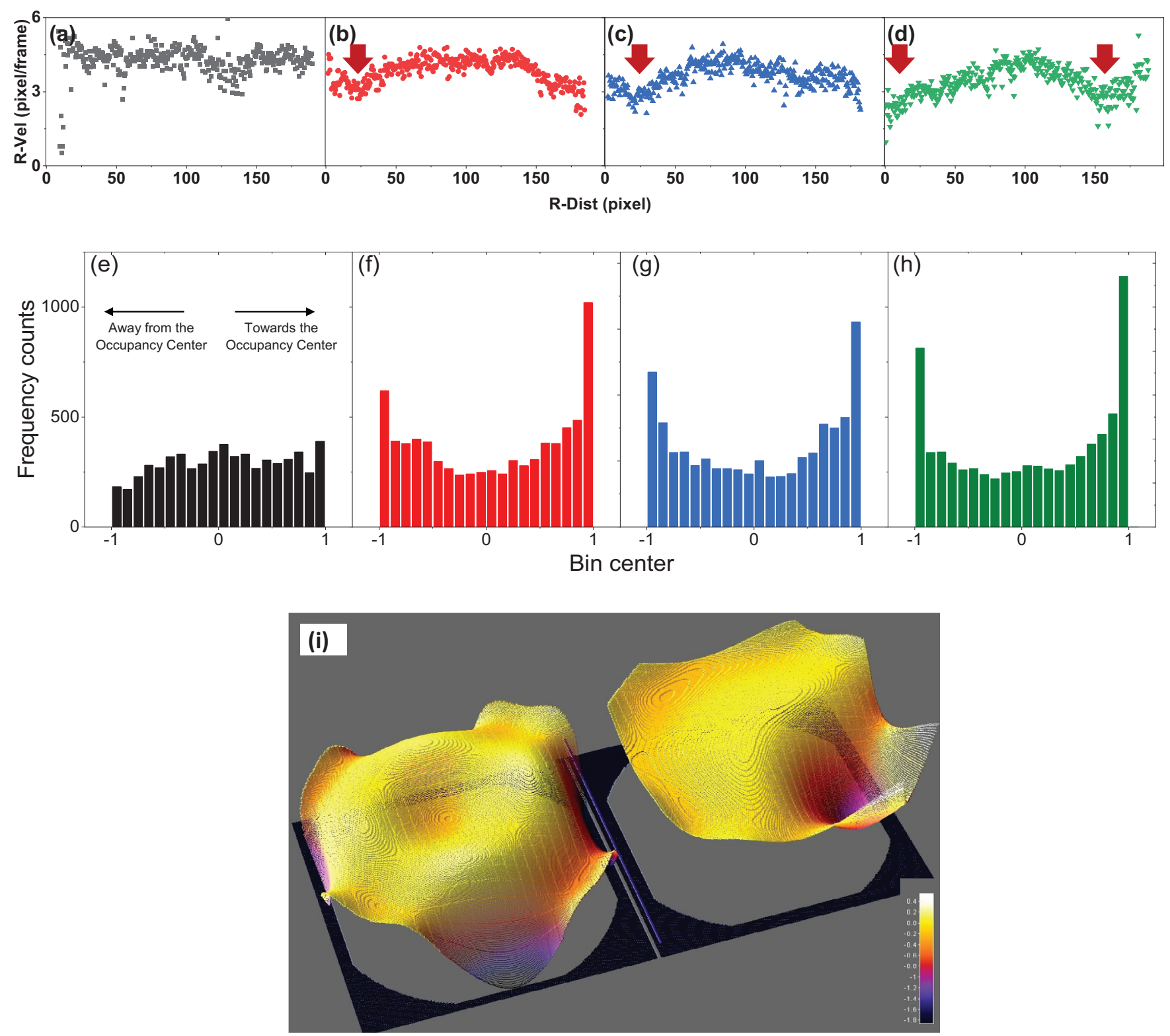

FIGURE 3 Divergence maps identify putative search centers or "sinks" as a result of differential distribution of velocity. (a-d) The solid shapes (circles, squares, and triangles) are the magnitude of velocity ( $\mathrm{R}$-Vel) measured as a function of radial distance (R-Dist) on training and probe days (D1, PD3, PD5, and PD7, respectively). On D1 the speed of the mice is invariant across space as the scatter plot is almost parallel to the $x$-axis representing the distance from the platform. With training, the speed of the mice is reduced near the platform and boundary $(r \rightarrow 0$ or $r \rightarrow 200$ pixels). R-Dist is in bins of 0.5 pixel and R-Vel represented as an average of all the times the animal traversed that bin. (e-h) Histogram (bin width of 0.1 ) representing the efficiency of movement toward occupancy center, given by $V_{\|} / V_{t}$, in Ptpn11 +/+ mice on D1, PD3, PD5, and PD7 is indicated as a function of training. The fraction of the movements oriented along the occupancy center increases as shown by the counts in bins -1 (aligned completely but away from occupancy center) and 1 (aligned completely toward the occupancy center). See Figure S8 for coarse bin equivalent. (i) Surface plot of divergence measure that is developed in theory generated using dataset Ptpn11 +/+ mice on day 1 (left) and on PD7 (right) shows that spatially uniform progressive reduction in speed leading to convergence peaks (negative divergence seen as valleys). Such convergence peaks are analogous to sinks in electro/fluid dynamics. On day 1 the divergence surface is largely invariant across the pool surface except for a mild depression at the center and at periphery of the pool. On probe day 7 , as the mice acquires the spatial memory for the platform location the surface changes its shape and has a clear valley centered around the platform location

that these mice focus their search away from both the platform location and the pool center, indicating that they lack a specific, localized spatial memory of the platform's location. However, on PD10 these mice do show signs of having acquired a specific spatial memory for the platform's location.
Additionally, the relative search diameter increases as a function of probe trials for both BALB/cJ and SWR/J mice (Figure $5 \mathrm{~g}$, BALB/cJ: Green diagonal bars, $\delta_{R S}=7 \pm 0.20$ [PD3], $\delta_{R S}=9 \pm 0.13$ [PD7], $\delta_{R S}=10 \pm 0.17$ [PD10] and Figure 5h, SWR/J: Blue diagonal bars, $\delta_{R S}=3.17 \pm 0.04$ [PD3], $\delta_{R S}=12.50 \pm 0.24$ [PD7], $\delta_{R S}=5 \pm 0.28$ [PD10]). With training, 


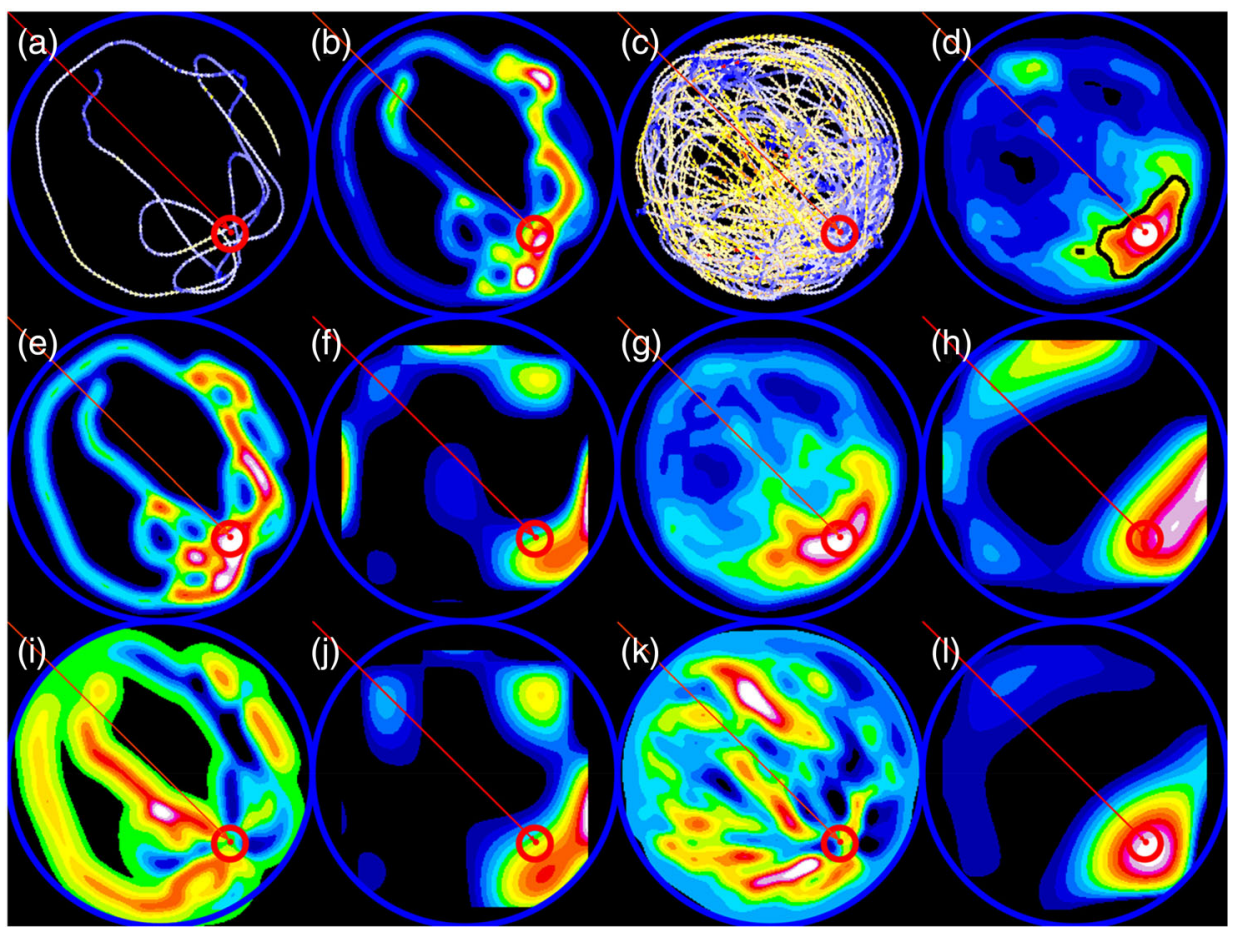

FIGURE 4 Component of the velocity along the occupancy center is better than the velocity at identifying putative search center. (a) and (c) show the velocity vector field maps for an individual Ptpn11+/+ mouse and a population of Ptpn11+/+ mice $(n=15)$ on PD7, respectively, LUT scale: 0-8 pixel/frame. (b) and (d) show the occupancy map for individual and population of mice. The most likely occupied region is demarcated as a black outline in (d). This region is identified based on maximum entropy segmentation for the population representation. Subsequently, the occupancy center is estimated and shown as a red arrow in both (b) and (d). (e) and (g) are the velocity magnitude heat maps for the individual mouse and population respectively. Divergence calculated in Cartesian coordinates is used to generate a map ( $\mathrm{f}$ and $\mathrm{h}$ ) and is placed inside the pool image. Bounding rectangle is used to orient and place the divergence map inside the pool. The generated velocity vector fields reveal several convergence hotspots as seen in $(f)$ and $(h)$. The cropped nature of the image is due to bounding rectangle imposed for performing polynomial surface fit. The bounding rectangle defines the region inside the pool where the mouse has traversed. In comparison, (i) and (k) represent the heat map of velocity vector component along the occupancy center (magnitude) for individual mouse and population, respectively. The divergence calculated as above, using these vector fields, are placed in the pool image and resultant images (j) and (I) show relatively fewer but prominent and localized convergence hotspots than (f) and (h). We term these convergence hotspots in velocity component maps as the putative search centers $\left(P_{c s}\right)$. We use the vector field representing the population of mice during a session in subsequent analysis for identifying the search center. Blue ROI marks the pool perimeter and red ROI marks the platform

their search strategies around the search center appear to shift from focused and localized searches to more diffused and generalized searches.

For $B A L B / c J$ mice, the search intensity is highest on the first probe trial (PD3; Figure 5i, Green hatched bars, $r l_{c s}=61 \% \pm 2 \%$ ). Combined with the accuracy value, these two measures suggest that the mice have learned the platform location and concentrate their searches in the correct location. However, on PD7, there is a reduction in the intensity of the searches $\left(r l_{c s}=25 \% \pm 0.4 \%\right)$. Since the accuracy of the search center is high (>90\%), the reduction in intensity could possibly be due to a memory of searching for the absent platform in PD3. The results suggest that the memory of the platform location is intact, but that the intention to locate it is reduced because there may be some acquired knowledge during PD3 that it may be absent from the pool. On PD10, the mice show an improvement in search intensity $\left(r l_{c s}=40 \% \pm 0.6 \%\right)$, potentially reflecting the effect of continued extended training between PD3 and PD10.

In SWR/J mice, the intensity of search improves from PD3 to PD10 (Figure $5 \mathrm{j}$, Blue hatched bars, $r l_{c s}=6.6 \% \pm 0.086 \%$ (PD3), $r l_{c s}=11.5 \% \pm 0.22 \%$ (PD7), and $r l_{c s}=59.3 \% \pm 3.38 \%$ (PD10). The extent of this search intention is poor $(<50 \%)$ for PD3 and PD7, reflecting poor spatial learning.

Thus, using convergence peaks as search centers, we can describe and compare the quality of spatial learning and memory across different probe trials for different strains of mice. From our comparisons, we find that the BALB/cJ mice perform better in the MWM than the SWR/J strain of mice.

\subsection{Ptpn11 ${ }^{\mathrm{D} 61 \mathrm{G} /+}$ mice learn the platform location with additional training as indicated by accuracy, uncertainty, and intensity of search}

Next, we analyzed the water maze performance of Ptpn11 $11^{\mathrm{D} 61 \mathrm{G} /+}$ mice, a model of NS (Araki et al., 2004). These mice have a gain-offunction point mutation in nonreceptor protein tyrosine phosphatase Ptpn11 gene found in Noonan patients (Araki et al., 2004, 2009), and they show learning and memory impairments, including spatial 

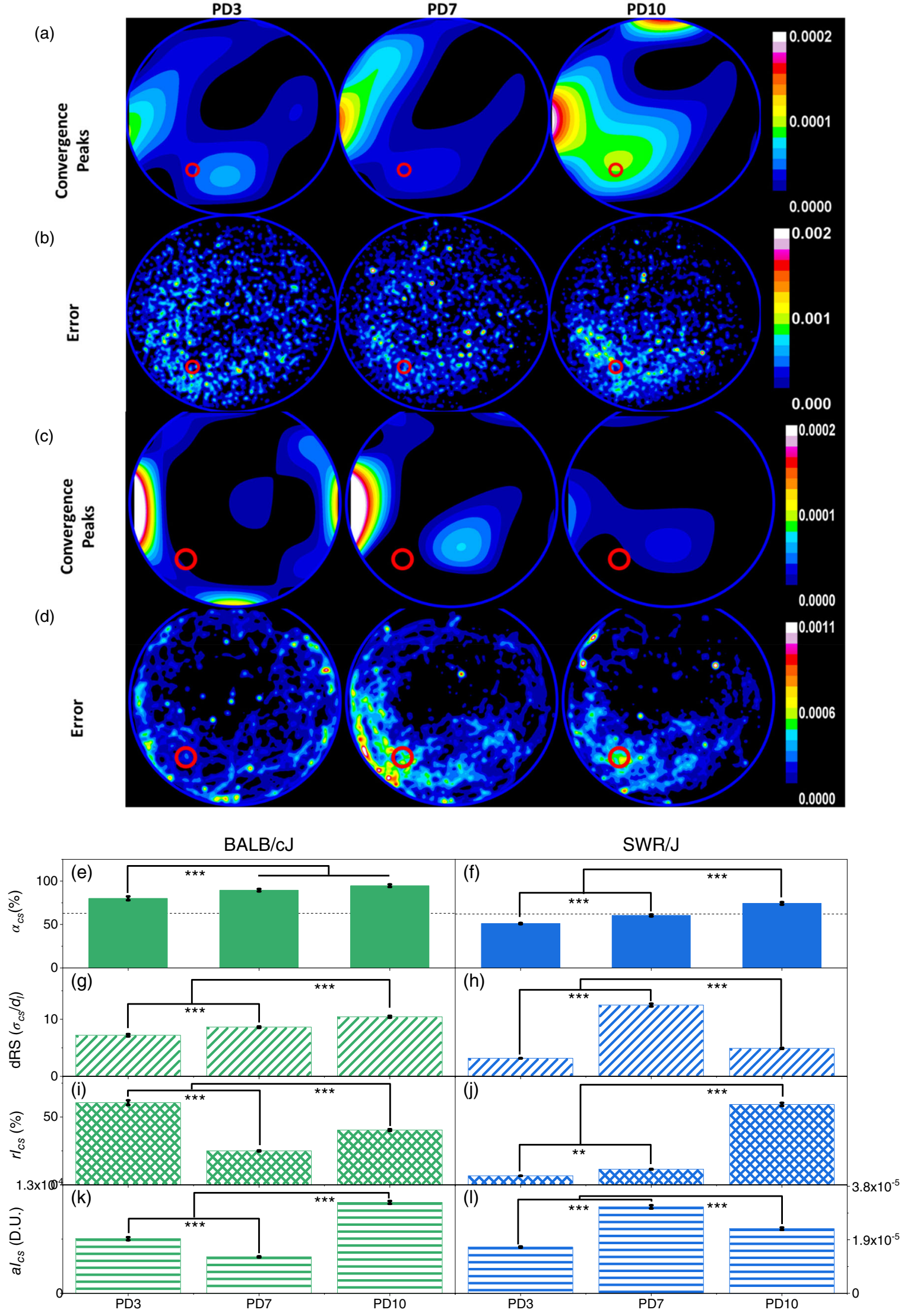

FIGURE 5 Legend on next page. 
learning and memory deficits in the MWM (Lee et al., 2014). Previous studies (Lee et al., 2014) showed that Ptpn11 ${ }^{\text {D61G/+ }}$ mice do not learn and remember the platform location after three training sessions with four trials each, as indicated by quadrant occupancy and proximity measure on the first probe trial (PD3). In contrast, with the same training WT littermates (Ptpn11 $11^{+/+}$mice) show clear evidence of having learned the platform's location. Additional training failed to improve the performance of Ptpn11 $11^{\mathrm{D} 61 \mathrm{G} /+}$ mice, as reported by quadrant occupancy and proximity measures on PD7. Our methods of analyses indicate that although Ptpn11 $101 \mathrm{G} /+$ mice did not show evidence that they had learned the platform location on PD3, additional training does improve their performance in the MWM: The divergence on velocity vector field along the occupancy center reveals a search center near the platform location on PD5 and PD7 (Figure 6c). Describing the convergence peak or search center in terms of accuracy of search, uncertainty about search, and intensity of search, reveals more clearly the quality of the spatial memory performance of Ptpn $11^{\mathrm{D} 61 \mathrm{G} /+}$ mice during probe trials. The accuracy of the search center to the platform location in Ptpn $11^{+/+}$mice is not statistically different across probe days (Figure 6e, Black solid bars, $\alpha=59 \% \pm 2.25 \%$ [D1], $\alpha=93 \%$ $\pm 2.41 \%$ [PD3], $\alpha=93 \% \pm 2.60 \%$ [PD5], $\alpha=96 \% \pm 1.34 \%$ [PD7]). Interestingly, the accuracy of the search center in Ptpn $11^{\mathrm{D} 61 \mathrm{G} /+}$ mice on PD5 is comparable to that of Ptpn11 $11^{++}$mice on PD3 (Figure 6f, Red solid bars, $\alpha=60 \% \pm 2.10 \%$ [D1], $\alpha=62 \% \pm 1.91 \%$ [PD3], $\alpha=87 \% \pm 1.57 \%$ [PD5], $\alpha=92 \% \pm 2.12 \%$ [PD7]; Two-way ANOVA for accuracy with genotype as between-subjects factor and probe day within-subjects factor, genotype $\times$ probe day interaction: $F_{3,92}=21$, $p<.001$; Bonferroni: Ptpn11 ${ }^{+/+}$PD3-Ptpn11 1 D61G/+ PD3: $p<.001$, Ptpn11 ${ }^{+/+}$PD3-Ptpn11 D61G/+ PD5: $p>$.5).

Additionally, the search intensity relative to the pool $\left(r l_{c s}\right)$ increases as a function of training. Ptpn $11^{\mathrm{D} 61 \mathrm{G} /+}$ mice increased their focused searches and their intention at search center on PD7, and these are similar to their WT littermates in this probe trial (Figure 6i,j). However, there is a significant difference in the absolute intensity of

FIGURE 5 Description of spatial memory in BALB/cJ and SWR/J strain of mice using the three parameters: Accuracy $(\alpha)$, uncertainty $\left(\delta_{R S}\right)$, and intensity of search $\left(I_{c s}\right)$. (a) and (c) are the divergence heat maps showing the convergence hotspots for the population of BALB/cJ ( $n=10$, a) and SWR/J ( $n=9-10, c)$ mice, respectively, on probe day 3 (PD3), probe day 7 (PD7), and probe day 10 (PD10). (b) and (d) are the error heat maps of the calculated divergence within the population of mice of BALB/cJ (b) and SWR/J (d) mice. A 2D Gaussian smoothing of radius $=5$ $(B A L B / c J)$ or $10(S W R / J)$ is used for visual representation. Pool perimeter is shown as a blue circle and the platform location is shown as red circle. (e) and (f) describe the accuracy of search center for BALB/cJ (e) and SWR/J (f). The accuracy in search increases to maximum asymptotically across probe trials for BALB/cJ mice (green solid bars, $\alpha=80 \% \pm 2.2 \%$ [PD3], $\alpha=91 \% \pm 1.3 \%$ [PD7], $\alpha=95 \% \pm 1.5 \%$ [PD10]) as expected. Comparatively, SWR/J mice show poor accuracy for the platform below chance level (defined in theory) on PD3 and at chance on PD7 (onesample $t$-test, PD3: $p<.001$, PD5: $p>.05)$ whereas it shows an improvement in accuracy $(p<.001)$ on PD10 (blue solid bars, $\alpha=51 \pm 0.66$ [PD3], $\alpha=61 \pm 1.15$ [PD7], $\alpha=74 \pm 1.41$ [PD10]). Dashed line shows the chance accuracy value (BALB/cJ: 63\%, SWR/J: 62\%). Two-way ANOVA with mice strain and training (measured across probe days) as factors revealed that accuracy is significantly different among the mice strain $\left(F_{1,52}=467, p<.001\right)$, and across training $\left(F_{2,52}=82, p<.001\right)$, however with a significant interaction between the mice strain and their training $\left(F_{2,52}=5.66, p<.01\right)$. Subsequent post hoc analysis indicated that BALB/cJ and SWR/J mice improved its accuracy over training (see table for pair-wise comparisons Table S2). Based on the difference in accuracy, we conclude that BALB/cJ are better learners than SWR/J mice. (g) and (h) describes the uncertainty in search center as relative search diameter $\left(\delta_{R S}\right)$ for BALB/cJ and SWR/J mice, respectively. The search strategy around the search center appears focused in nature on PD3 for BALB/cJ (green diagonal bar, $\delta_{R S}=7 \pm 0.20$ ). In case of the SWR/J mice, although the search center is inaccurate, its search is focused (blue diagonal bar, $\delta_{R S}=3.17 \pm 0.04$ ). The relative search diameter increases on subsequent probe trials, indicating that both strains of mice use a more diffused search strategy in later probe trials (BALB/cJ: Green diagonal bars, $\delta_{R S}=9$ \pm 0.13 [PD7], $\delta_{R S}=10 \pm 0.17$ [PD10] and SWR/J: Blue diagonal bars, $\delta_{R S}=12.50 \pm 0.24$ [PD7], $\delta_{R S}=5 \pm 0.28$ [PD10]). Two-way ANOVA for uncertainty with mice strain and training showed that the means of the strains are significantly different $\left(F_{1,52}=217, p<.001\right)$, training had an effect $\left(F_{2,52}=586, p<.001\right)$ however the effect of training is different among the strains as there is an interaction $\left(F_{2,52}=504, p<.001\right)$ between these factors. Post hoc analysis revealed that the search diameter shows a differential change as function of training (see Table S3 for pair-wise comparisons). (i) and (j) represents the relative search intensity $\left(r l_{c s}\right)$ for BALB/cJ and SWR/J mice. Since the relative intensity of search is obtained from normalizing the absolute intensity at search center with the maximum intensity observed during the session, we can compare the relative intensity across datasets that have been sampled at different frame rates. In BALB/cJ mice, the relative search intensity on the PD3 is the highest (green hatched bars, $r l_{c s}=61 \% \pm 2 \%$ ). However, on PD7, the intensity of search reduces $\left(r l_{c s}=25 \% \pm 0.4 \%\right)$ possibly due to effect of extinction but recovers and increases at PD10 $\left(r l_{c S}=40 \% \pm 0.6 \%\right)$. In SWR/J mice, the intensity of search improves with probe trials (blue hatched bars, $r l_{c s}=6.6 \% \pm 0.086 \%$ [PD3], $r l_{c s}=11.5 \% \pm 0.22 \%$ [PD7], $r l_{c s}=59.3 \% \pm 3.38 \%$ [PD10]) though the overall extent of this search intention is poor (<50\%). Two-way ANOVA for relative search intensity with strain and training as factors showed that the search intensity significantly differed both as function of strain $\left(F_{1,52}=488, p<.001\right)$ as well as training $\left(F_{2,52}=608, p<.001\right)$ with a strong interaction $\left(F_{2,52}=835, p<.001\right)$. Post hoc analysis (see Table S4 for pair-wise comparisons) substantiated our interpretation stated above. (k) represents the absolute search intensity $\left(a l_{c s}\right)$ for BALB/cJ on PD3, PD7, and P10. In BALB/cJ mice, the intensity of search is given in green horizontal bars, $a l_{c s}=6.5 \mathrm{E}-5$ $\pm 1.8 \mathrm{E}-6$ (PD3), $a l_{c s}=4.4 \mathrm{E}-5 \pm 6.6 \mathrm{E}-7$ (PD7), $a l_{c s}=1.1 \mathrm{E}-4 \pm 1.7 \mathrm{E}-6$ (PD10). One-way ANOVA for absolute search intensity showed the means of the absolute search intensity are significantly different $\left(F_{2,27}=488, p<.001\right)$. Post hoc analysis established that the differences between the probe trail days are significant (see Table S5 for pair-wise comparisons). (I) represents the absolute search intensity ( $a I_{c S}$ ) for SWR/J mice on PD3, PD7, and P10. In SWR/J mice, the intensity of search improves with probe trials (blue horizontal bars, al $c s=1.64 \mathrm{E}-5 \pm 2.13 \mathrm{E}-7$ [PD3], $a l_{c s}=3.07 \mathrm{E}-5 \pm 1.749 \mathrm{E}-6$ [PD7],$a l_{c s}=2.29 \mathrm{E}-5 \pm 4.36 \mathrm{E}-7$ [PD10]). One-way ANOVA for absolute search intensity showed the means of the absolute search intensity are significantly different $\left(F_{2,25}=286.7677, p<.001\right)$. Post hoc analysis established that the progressive increase seen across the probe trial days is significant (see Table S5 for pair-wise comparisons) 
search $\left(a l_{c s}\right.$ ) between Ptpn11 $1^{+/+}$and Ptpn11 ${ }^{\mathrm{D} 61 \mathrm{G} /+}$ mice (Figure 6k,l). Absolute intensity at a location in a divergence image measures the extent by which vector decreases over a unit distance. Given that in our case the video is uniformly sampled, this measure directly reflects a decrease observed over time, and hence the velocity. On the other hand, relative intensity measures the fraction of slow down with respect to the steepest decline observed in the pool. Thus, the differences in search intensities $\left(a l_{c s}\right)$ could reflect either cognitive or motor deficits, while $r_{c s}$ differences reflect differences in spatial learning and memory. The difference we observe in search intensities $\left(a l_{c s}\right)$ in WT and mutants could be due to the differences in their swimming ability. In contrast, $r l_{c s}$, accuracy and uncertainty are sensitive to deficits in spatial learning and memory and are not directly affected by differences in motor performance.
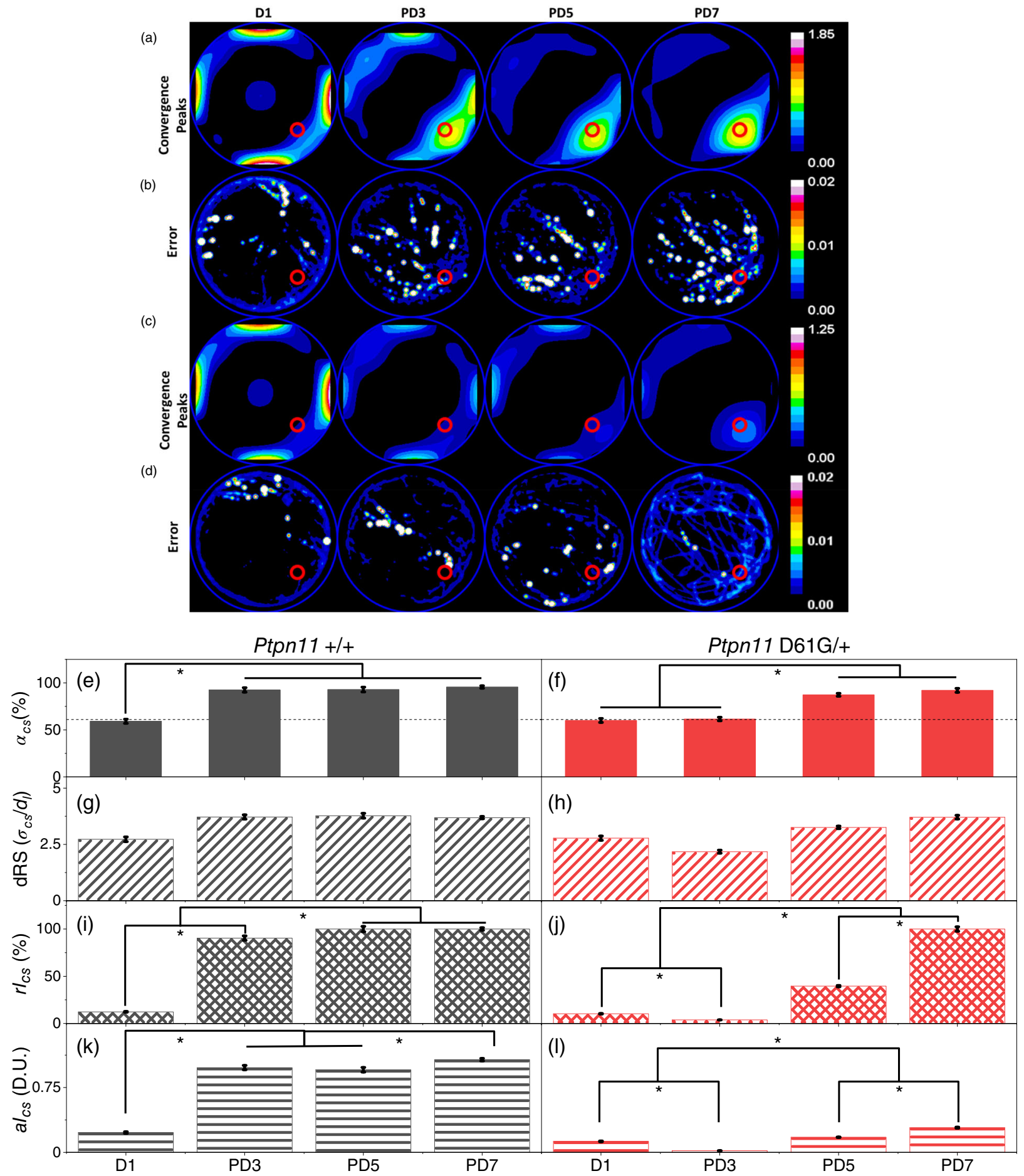

FIGURE 6 Legend on next page. 
To further test the increased sensitivity of our methods and reduce the effect of motor impairment characteristic of NS (Lee et al., 2014; Romano et al., 2010), we a ran another set of analyses with a smaller number of animals in which the NS mutation was restricted to the dorsal hippocampus (HPC), a region related to spatial memory processing (Andersen et al., 2009). Previous results showed that the adeno-associated virus induced expression of the PTPN11 ${ }^{\mathrm{D} 61 \mathrm{G}}$ allele (AAV-PTPN11 ${ }^{\mathrm{D} 61 \mathrm{G}}$ ) only in the HPC leads to spatial memory deficits similar to transgenic animals, including in the
MWM (Lee et al., 2014). The Figure S6a,b show the convergence map generated for this set of mice at D1, PD5, PD7, and PD11. Subsequent measures of accuracy (Figure S6c,d), uncertainty (Figure S6e,f), and relative intensity of search (Figure S6g,h) show clear evidence for learning induced behavioral changes, while the residence time measure (Figure S6i,j) of this subset does not show any discernible change suggesting that statistical power of residence time measure is substantially lower and requires a greater number of mice to be used for detecting these subtle changes.

FIGURE 6 Description of MWM spatial memory in Ptpn11 D61G/+ and wild type litters mates (Ptpn11 +/+) using the three parameters accuracy $(\alpha)$, uncertainty $\left(\delta_{R S}\right)$, and intensity of search $\left(I_{c S}\right)$ show that with additional training D61G/+ mice recall the platform location with accuracy and relative search intensity comparable to wild type littermates (PD3). (a) and (c) are the divergence heat maps showing the convergence hotspots for the population of Ptpn11 +/+ wild type (WT, $n=15)$ and Ptpn11 D61G/ + transgenic (D61G, $n=10)$ mice, respectively, on training day 1 (D1), probe day 3 (PD3), probe day 5 (PD5) and probe day 7 (PD7). D1 heat map serves as a representation of the surface when the mouse has no knowledge of the platform location. After three training sessions of four trials each, WT mice show a convergence peak close to the platform location in the first probe trial on PD3, whereas the D61G/+ mice do not show any peak near the platform. With additional training, D61G show a convergence peak adjacent to the platform location on PD5 and PD7 indicating the presence of spatial memory. (b) and (d) is a visual representation of the error estimates of calculated divergence within a population of mice for Ptpn11+/+ and Ptpn11 D61G/+ mice respectively. A Gaussian blur of radius 2 was applied for visual representation. Pool perimeter is shown as a blue circle and the platform location is shown as red circle. (e) and (f) quantify the accuracy of search center. In both wildtype and mutant mice, the accuracy of search center starts at a chance level (61\%, dashed line) on D1 (WT: $\alpha=59 \pm 2.25$ [D1, black solid bars]; one-sample $t$-test, $p>.05$ and D61G/ $+: \alpha=60 \pm 2.10$ [D1, red solid bar]; one-sample $t$-test $p>.05)$. In WT mice the accuracy reaches a maximum on PD3 itself ( $\alpha=93 \pm 2.41$ [PD3], $\alpha=93 \pm 2.60$ [PD5], $\alpha=96 \pm 1.34$ [PD7]). We see that D61G/ + mice perform at chance level when tested on PD3 ( $\alpha=62 \pm 1.91$ [PD3, red solid bar]; one-sample t-test, $p>$.05). However, with additional training, the accuracy of search center improves on PD5 and PD7 (red solid bars $\alpha=87 \pm 1.57$ [PD5], $\alpha=92 \pm 2.12$ [PD7]; Two-way ANOVA for accuracy with genotype and training as factors showed that the means across the genotype $\left(F_{1,92}=39, p<.001\right)$, as well as across training day $\left(F_{3,92}=97, p<.001\right)$ are different with a significant interaction $\left(F_{3,92}=21, p<.001\right)$. Post hoc analysis (see Table S6 for pair-wise comparison) are consistent with our interpretation provided above. Interestingly, we see that the accuracy of TG mice on PD5 is comparable to that of WT mice on PD3 (Bonferroni: WT PD3-D61G/+ PD3: $p<.001$, WT PD3-D61G/+ PD5: $p>.5$ ) indicating that $\mathrm{D} 61 \mathrm{G} /+$ mice are able to learn the spatial memory task despite their cognitive deficits however with a slow rate of acquisition. $(\mathrm{g})$ and $(\mathrm{h})$ quantifies the uncertainty in search center in terms of relative search diameter $\left(\delta_{R S}\right)$ for WT and D61G/+ mice. In both WT (black diagonal bars, $\delta_{R S}=2.7 \pm 0.103$ [D1], $\delta_{R S}=3.7 \pm 0.097$ [PD3], $\delta_{R S}=3.7 \pm 0.106$ [PD5], $\delta_{R S}=3.7 \pm 0.052$ [PD7]) and D61G/ + (red diagonal bars, $\delta_{R S}=2.7 \pm 0.097$ [D1], $\delta_{R S}=2.18 \pm 0.067$ [PD3], $\delta_{R S}=3.3 \pm 0.059$ [PD5], $\delta_{R S}=3.7 \pm 0.085$ [PD7]) mice, the relative search diameter was found to be comparable across the three probe trials. Two-way ANOVA for uncertainty in search with genotype and training as factors showed that the difference in means across both, the genotype $\left(F_{1,92}=1.78, p>.05\right)$ and across training day $\left(F_{3,92}=1.47, p>.05\right)$, is not statistically significant. The interaction between the genotype and training day was also not statistically significant $\left(F_{3,92}=0.996, p>.05\right.$, see Table $\left.S 7\right)$. This indicates that the search area or focus does not change across probe trials. (i) and (j) quantifies the relative intensity of search ( $\left.r l_{c s}\right)$ for $W T$ and D61G/ + mice. In case of WT mice, the relative intensity of search at the search center $\left(P_{c s}\right)$ is high on PD3 itself and reaches a maximum on PD5 and PD7 (black hatched bars, $r l_{c s}=12 \pm 1$ [D1], $r l_{c s}=90 \pm 9$ [PD3], $r l_{c s}=100 \pm 11$ [PD5], $r l_{c s}=100 \pm 5$ [PD7]). In D61G/+ mice, the intensity of search at $P_{c s}$ steadily improves from a low value on PD3 to a maximum on PD7 (red hatched bars, $r l_{c s}=10 \pm 1$ [D1], $r l_{c s}=3.8 \pm 0.26$ [PD3], $r l_{c s}=40 \pm 2$ [PD5], $r l_{c s}=100 \pm 9$ [PD7]). Two-way ANOVA for relative intensity with genotype and training as factors show that the means are significantly different both as a function of genotype $\left(F_{1,92}=1.3 \mathrm{E} 7, p<.001\right)$ and training day $\left(F_{3,92}=31.3 \mathrm{E} 7, p<.001\right)$. The interaction between the two factors was also statistically significant $\left(F_{3,92}=4.5 \mathrm{E} 6, p<.001\right)$. Post hoc analysis shows that the WT mice learned to search intently on PD3 (D1 < PD3) even though PD5 and PD7 search intensities are higher (PD3 < PD5, PD7) while the mutant mice progressively increased its search intensity from only after PD3 (D1 PD3), that is intensity of search at PD3 < PD5 < PD7 as seen from pairwise comparisons in Table S8. The evidence further supports the notion that D61G/+ mutant mice learn the platform location and contribute its effort at the search center after intense training. $(\mathrm{k})$ and $(\mathrm{I})$ quantifies the absolute intensity of search $\left(a l_{c s}\right)$ for WT and D61G/ + mice. The absolute intensity of search at $P_{c s}$ is significantly different on probe days compared with training day in WT mice (black horizontal bars, $a_{c s}=0.229 \pm 0.0087$ [D1], al ${ }_{c s}=0.98$ \pm 0.02548 [PD3], $a l_{c s}=0.955 \pm 0.02674$ [PD5], $a l_{c s}=1.072 \pm 0.01501$ [PD7]). In D61G/ + mice, the absolute intensity of search at $P_{c s}$ steadily improves with training (red horizontal bars, $a l_{c s}=0.128 \pm 0.00448$ [D1], $a l_{c s}=0.019 \pm 5.89 \mathrm{E}-4$ [PD3], $a l_{c s}=0.175 \pm 0.00315$ [PD5], $a l_{c s}=0.285$ \pm 0.00656 [PD7]). Both D61G as well as their littermates are trained and probed on the same experimental setup (with experimenter being blind to the genotype) as a result the absolute intensities can be compared across genotypes. Two-way ANOVA for comparing absolute intensity across genotypes and training as two factors indicate that absolute search intensities are significantly different between WT and D61G mice $\left(F_{1,92}=2677, p<.001\right)$ and as a function of training they increase $\left(F_{3,92}=285, p<.001\right)$ their intention of search though at different rates (genotype $\times$ probe day interaction: $F_{3,92}=224, p<.001$ ). See Table S9 for pair-wise comparison. This we interpret as resulting from possible motor as well as cognitive deficits 


\section{5 | The spatial learning and memory deficit of Ptpn11 ${ }^{\mathrm{N} 308 \mathrm{D} /+}$ mice in the MWM is rescued by SL327}

Next, we analyzed the MWM performance of another mouse model of NS (Ptpn11 $\left.{ }^{\mathrm{N} 308 \mathrm{D} /+}\right)$. Ptpn11 $1^{\mathrm{N} 308 \mathrm{D} /+}$ mice have an heterozygous knock-in gain-of-function mutation found in some Noonan patients (Araki et al., 2004, 2009) and their spatial learning and memory deficits are known to be milder than those in Ptpn11 1 D61G/+ mice (Lee et al., 2014). While Ptpn $11^{\mathrm{N} 308 \mathrm{D} /+}$ mice show similar performance to WT littermates as measured by latency to reach the platform during training, during a probe trial after 3 days of training (PD3), these mice spend significantly less time in the target quadrant than their WT littermates. Ptpn11 ${ }^{\mathrm{N} 308 \mathrm{D} /+}$ mice also search farther away from the platform, as shown by the proximity measure. However, with two additional days of training, these mice show comparable spatial learning and memory performance in PD5, as measured using quadrant (Figure S4aa-ad) and proximity measures (Figure S5i).
We analyzed the swim trajectories of Ptpn11 ${ }^{\mathrm{N} 308 \mathrm{D} /+}$ and their WT littermates on PD5. Additionally, we investigated whether SL327, a MEK inhibitor, previously shown to reverse the memory deficits of Ptpn11 ${ }^{\mathrm{D} 61 \mathrm{G} /+}$ mice (Lee et al., 2014), could also improve the accuracy and uncertainty in search of the platform location in Ptpn11 N308D/+ mice. First, we found that there are subtle but significant differences in spatial learning and memory performance in terms of accuracy, uncertainty, and intensity of search between $\operatorname{Ptpn} 11^{\mathrm{N} 308 \mathrm{D} /+}$ and their WT littermates. Ptpn $11^{\mathrm{N} 308 \mathrm{D} /+}$ showed an accuracy of $80 \% \pm 2 \%$ with an uncertainty of $5.5 \pm 0.16$ relative search diameter, whereas the WT mice showed a superior accuracy of $94 \% \pm 2 \%$ with an uncertainty of $4.01 \pm 0.092$ in relative search diameters (refer to Tables S10 and S11 for statistical comparison). After treatment with SL327, Ptpn11 ${ }^{\mathrm{N} 308 \mathrm{D} /+}$ improved their accuracy to $95 \% \pm 2 \%$ with a relative search diameter of $4.33 \pm 0.074$ (Tables S10 and S11) Incidentally, treating WT mice with SL327 has the opposite effect in their accuracy and search area, that is, $\alpha_{c S}=88 \% \pm 4 \%$ and $\delta_{R S}=5.22$

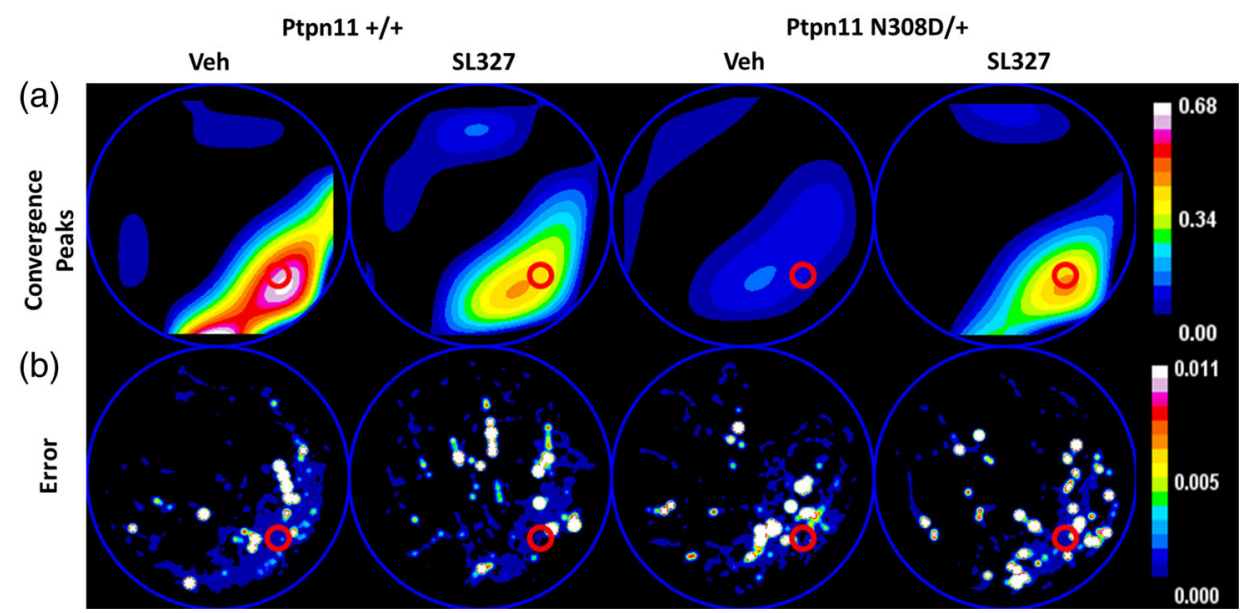

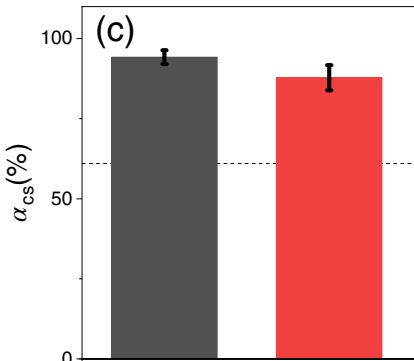

(e)

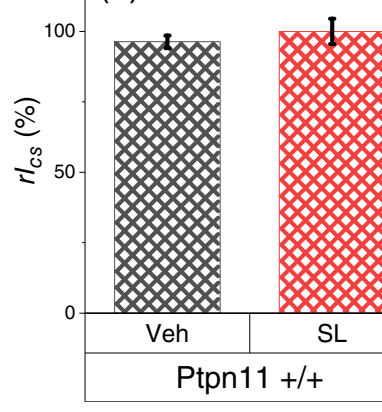

(d)
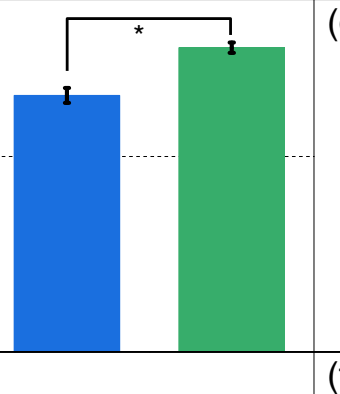

(f)

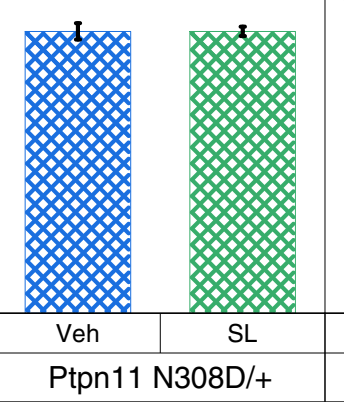

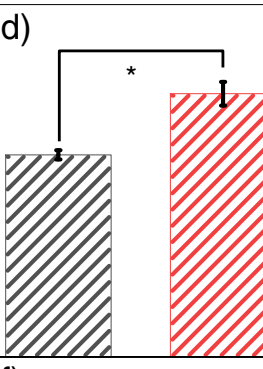

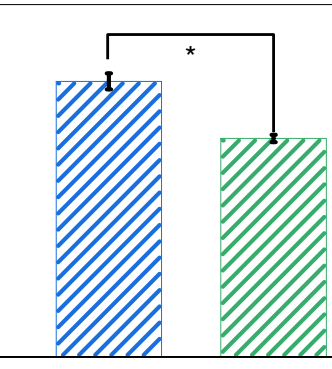


\pm 0.235 (Figure 7c-e), Two-way ANOVA for accuracy with genotype as between-subjects factor and treatment within-subjects factor, genotype $\times$ treatment: $F_{1,38}=15, p<.001$; Bonferroni: Ptpn11 N308D/+ Veh-Ptpn11 N308D/+ SL $p<.01$, Ptpn11 N308D/+ Veh-Ptpn11 +/+ Veh: $p<.01)$. These results are consistent with our interpretation that that there is an impairment in spatial memory in Ptpn11 ${ }^{\mathrm{N} 308 \mathrm{D} /+}$ mice and that the MEK inhibitor SL327 is capable of rescuing this deficit even though it causes a mild decline in performance in WT littermates, a compelling demonstration of the specificity of this drug treatment.

\section{6 | Tracking performance in a goal reversal task in the MWM}

Finally, we analyzed a publicly available dataset with two strains of mice (10-week old female mice C57BI/6J and DBA/2J) tested in a goal reversal task (Overall et al., 2020). These analyses highlight the power of the current vector field property-based measures in detecting search centers. In the goal reversal task, the mice are thought to acquire a memory for the old as well as for the new platform locations, and due to a recency effect, they bias their search toward the new platform location.

Previous results showed that $\mathrm{DBA} / 2 \mathrm{~J}$ mice perform poorly in hippocampal-dependent tasks, including in the MWM (Paylor et al., 1994; Upchurch \& Wehner, 1988). Indeed, our analyses revealed that $\mathrm{DBA} / 2 \mathrm{~J}$ mice do not show the presence of a specific search center on PD3 (Figure 8c). In comparison, analyses of PD3 revealed that $\mathrm{C} 57 \mathrm{BI} / 6 \mathrm{~J}$ mice learn the platform location with an accuracy of $86 \% \pm 3 \%$, relative search diameter of $4.4 \pm 0.15$, and relative search intensity of $100 \% \pm 3 \%$ (Figure $8 a, e, g, i)$.

As predicted, after being trained on two platform locations, analyses of the mice's performance with our methods reveals the presence of two convergence peaks or search centers on probe day 5 (PD5). The two search centers correspond to the presumed memories for the target location (i.e., the platform location in north-east quadrant on training days 3 and 4), as well as memory for the conflict location (i.e., the platform in the south-west quadrant on training on days 1 and 2; Figure 8a-d). Quantifying the search centers in terms of accuracy, uncertainty and intensity of search reveals differences in the nature of the two memories.

FIGURE 7 Accuracy and uncertainty are able to detect the rescuing of spatial memory deficits seen in Ptpn11 N308D/+ mice by the administration of SL327. (a) Divergence heat maps representing the convergence peaks for Ptpn11+/+ WT mice $(n=11)$ and Ptpn11 N308D/+ mutant mice $(n=10)$ for saline (veh) and drug (SL327) treated conditions. (b) Heat map of the error estimated on divergence values indicates the variation within the population of mice. A Gaussian blur (radius =6) was applied for visualizing the error in the sampled pixels. (c) Accuracy of search center reveals that Ptpn11 +/+ and Ptpn11 N30D/+ show subtle difference in spatial memory (solid bars: Ptpn11 +/+ Veh $\alpha_{c s}=94 \%$ $\pm 2 \%$ [black], Ptpn11 N308D/+ Veh $\alpha_{c s}=80 \% \pm 2 \%$ [red]). Treatment with MEK inhibitor SL327 rescues the deficit and brings the performance of N308D/+ mutant to the level of WT (Ptpn11 +/ SL327 $\alpha_{c s}=88 \pm 4$ [blue], Ptpn11 N308D/+ SL327 $\alpha_{c s}=95 \% \pm 2 \%$ [green]; Bonferroni: Ptpn11 N308D/+ Veh-Ptpn11 N308D/+ SL: $p<.01$, Ptpn11 N308D/+ Veh-Ptpn11 +/+ Veh: $p<.01)$. Two-way ANOVA for accuracy with genotype and treatment as two factors established that although there is no difference in the means of genotype $\left(F_{1,38}=1.6, p>.05\right)$ and the treatment $\left(F_{1,38}=2.4, p>.05\right)$ however there was significant interaction $\left(F_{1,38}=15, p<.001\right)$ indicating that the means of these factors are changing in opposite directions as revealed by the post hoc analysis with the rescue due to SL327 is significantly higher than the decrement in accuracy seen in the wild type following the administration of SL327 (see Table S10 for pair-wise comparisons). Dashed line shows the chance accuracy value (61\%). (d) Uncertainty in spatial memory is statistically different between N308D/+ mutant and WT littermates (diagonal bars: Ptpn11 +/+ Veh $\delta_{\text {RS }}=4.01 \pm 0.092$ [black], Ptpn11 N308D $/+$ Veh $\delta_{R S}=5.47 \pm 0.159$ [red]; Bonferroni: Ptpn11 N308D $/+$ Veh-Ptpn11 +/ + Veh: $p<.001$ ). Administration of SL327 makes the search focused for the mutant (Ptpn11 N308D/+ SL327 $\delta_{R S}=4.33 \pm 0.074$ [green]), however it also increases the search diameter in the wild type I littermates (Ptpn11 $+/+$ SL327 $\delta_{R S}=5.22 \pm 0.235$ [blue]). N308D/+ mutant mice improve their performance (Bonferroni: Ptpn11 N308D/+ Veh-Ptpn11 N308D/+ SL, $p<.001$ ), whereas the search area of their wildtype littermates becomes more diffused. Two-way ANOVA for uncertainty performed with genotype and treatment showed significant interaction (genotype $x$ treatment interaction: $F_{1,38}=56, p<.001$ ), without any main effect (genotype: $F_{1,38}=3.3, p>.05$, treatment: $F_{1,38}=0.068, p<.05$ ). See Table S11 for pair-wise comparison. (e) The relative search intensities at the search center are similar in both strains of mice (hatched bars: Ptpn11 +/ + Veh $r_{c s}=96 \pm 2$ [black], Ptpn11 N308D/+ Veh $r l_{c s}=100 \pm 3$ [red]). Treatment of SL327 did not alter the relative intensity contributed to the search center in case of both strains of mice (Ptpn11 $+/+\mathrm{SL} 327 \mathrm{rl}_{c S}=100 \pm 5$ [blue], Ptpn11 N308D/ + SL327 rl $l_{c s}=100 \pm 2$ [green]). Two-way ANOVA for relative search intensity with genotype and treatment as two factors found no significant difference among the means nor any significant interaction between the factors (genotype: $F_{1,38}=0.36, p>.05$, treatment: $F_{1,38}=0.36, p>.05$, genotype $\times$ treatment interaction: $F_{1,38}=0.36, p>.05$. See Table S12). (f) The absolute intensities measured from these two groups of mice share the same units as they are carried out on the same setup at the same frame rate. The intensity of search differs in case of Ptpn11 N308D and its WT littermates (horizontal bars: Ptpn11 +/ + Veh al $c$ $=0.65 \pm 0.015$ [black], Ptpn11 N308D/+ Veh $a l_{c s}=0.13 \pm 0.004$ [red]). Treatment of SL327 rescues the deficit seen in N308D mice such that the treated mice search more intently than that of the vehicle treated mutant mice. Although SL327 affects the performance of the WT type mice but the direction of change is opposite to that of the N308D mutant. (Ptpn11 $+/+$ SL327 al $c$ $=0.43$ \pm 0.019 [blue], Ptpn11 N308D/+ SL327 al $c$ = $0.44 \pm 0.007$ [green]; Bonferroni: Ptpn11 N308D/+ SL-Ptpn11 +/+ SL, $p>.5$ ). Two-way ANOVA done on the absolute search intensity with genotype and treatment as two factors showed that means of both the factors (genotype: $F_{1,38}=1749, p<.001$, day: $\left.F_{1,38}=55, p<.001\right)$, as well as interaction is significantly different $\left(F_{1,38}=1217, p<.001\right)$. Post hoc analysis (Table S13) confirms our earlier interpretation that SL327 rescues the spatial memory deficit seen in N308D mice although the treated group search intention is still significantly lesser than the wild type vehicle. We also note that administration of SL327 significantly lowers the search intention in the wild type animals 
Interestingly, we did not detect a difference in accuracy of the search centers to their respective platform positions (i.e., search center near the target location [Peak\#1 new] and search center near the conflict location [Peak\#2 old]). We conclude that since the mice recall the old as well as the new platform locations with similar accuracy, the strength of the memory for both locations is comparatively high (Figure 8e,f). Thus, learning the second location does not seem to significantly weaken the memory for the first location.

In $\mathrm{C} 57 \mathrm{BI} / 6 \mathrm{~J}$ mice, the relative intensity of the search $\left(r l_{c s}\right)$ at the search center corresponding to the new platform location (Peak\#1 new), is significantly higher than the relative intensity of search corresponding to the old platform location (Peak\#2 old; Figure 8i), indicating that a possible recency effect for the new platform location biases the mice to search more intensely in the target location. However, when the mice fail to find the platform at the target location, they also investigate the other (i.e., conflict) location. Nevertheless, the mice spend considerably less time searching in the conflict location. Similarly, the relative intensity of search $\left(r l_{c s}\right)$ at the search center corresponding to the new platform location (Peak\#1 new), is slightly higher than the relative intensity of search corresponding to the old platform location in DBA/2J mice (Peak\#1 old; Figure 8j). We infer that the DBA/2J mice search at both platform locations, though they prefer to search at the new platform location slightly more intensely. Similar to $C 57 \mathrm{BI} / 6 \mathrm{~J}$, the $\mathrm{DBA} / 2 \mathrm{~J}$ mice search at the most recent training location, although they never truly learnt the first platform location.

Since the purpose of this study was to determine whether training resulted in goal reversal, we defined a goal reversal efficiency measure (GRE) as $\mathrm{rl}_{c s}$ at the new location subtracted by the $r l_{c s}$ at the old platform location divided by the sum of $r l_{c s}$ at both

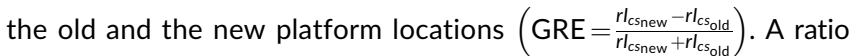
of 1 in this measure would indicate goal reversal, while -1 would indicate no reversal, and 0 would mean no learning. We find the goal reversal efficiency for $\mathrm{C} 57 \mathrm{BI} / 6 \mathrm{~J}$ to be $\sim 0.78$ and $\mathrm{DBA} / 2 \mathrm{~J}$ to be $\sim 0.15$, indicating that $\mathrm{C} 57 \mathrm{BI} / 6 \mathrm{~J}$ are more adapt at goal reversal than DBA/2J mice.
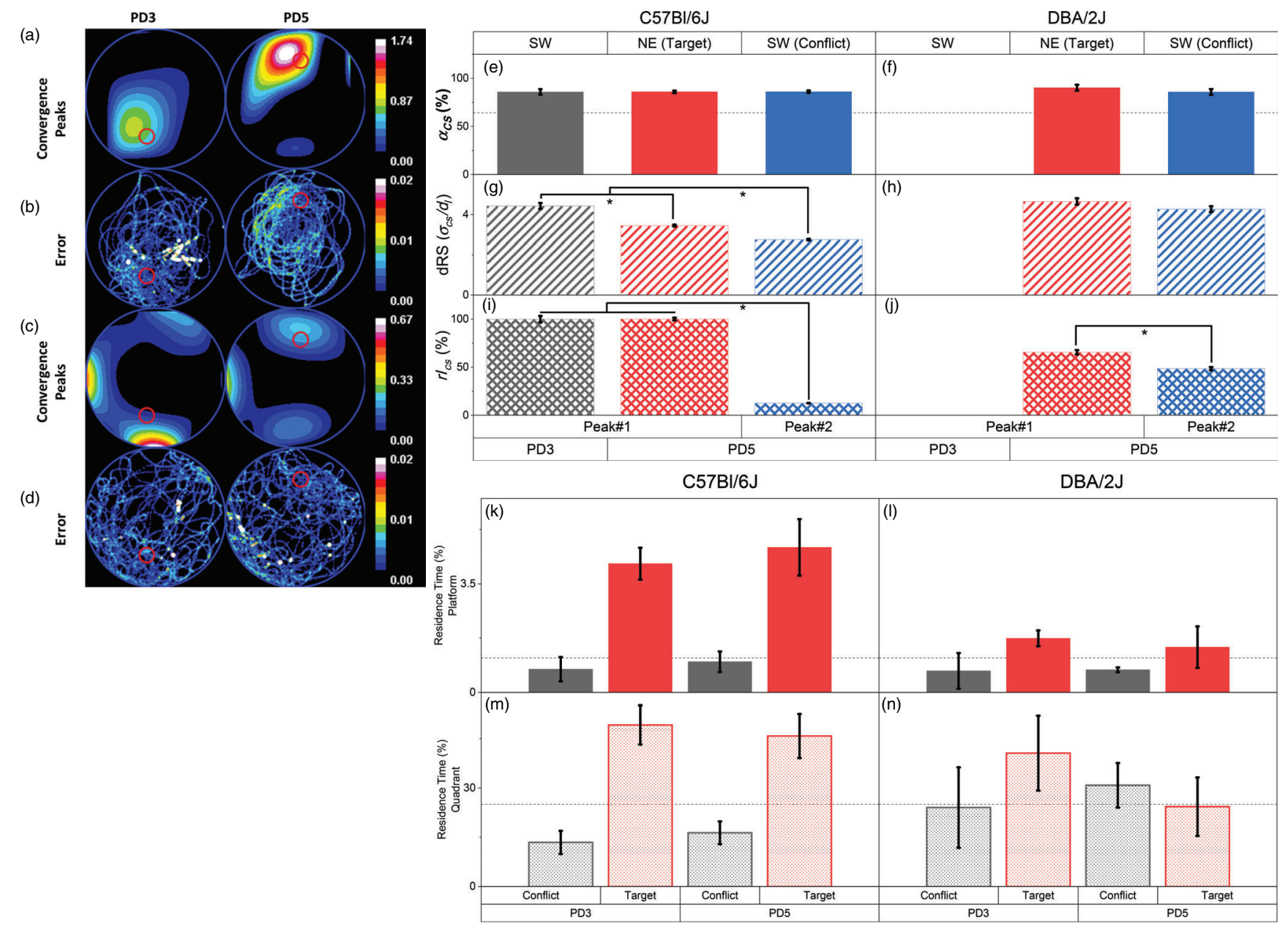

FIGURE 8 Legend on next page. 


\section{4 | DISCUSSION}

Here, we introduce a new set of informative measures to describe spatial learning and memory in a navigational task based on timestamped $x y$ trajectories. The use of velocity-based vector fields and their properties allows the pinpointing of putative search centers in the MWM. With these search centers, it is possible to describe spatial memory using three novel metrics: accuracy of search center to the target location, uncertainty of the search or spread in search area, and the intensity or extent of effort in search.
Analyzing swim trajectories using the proposed metrics enabled us to detect subtle memory deficits in four very different MWM datasets. We show a difference in the strength of spatial learning and memory for two different mice strains subjected to the same training paradigm (BALB/CJ and SWR/J). We also analyzed two different mouse models of NS, including Ptpn $11^{\mathrm{D} 61 \mathrm{G} /+}$ mice that were previously thought to lack spatial learning and memory even after extended training. Our analyses revealed that Ptpn $11^{\mathrm{D} 61 \mathrm{G} /+}$ mice do indeed show evidence of learning the platform location after extended training, and that their accuracy and uncertainty in the

FIGURE 8 Convergence hotspots detects the memory for old as well as new platform location in goal reversal task. C57BI/6J and DBA/2J strains of mice were trained with the platform in the south-west (SW) quadrant on probe day 3 (PD3), whereas the platform was shifted to the north-east (NE) quadrant on probe day 5 (PD5). Using accuracy $(\alpha)$, uncertainty $\left(\delta_{R S}\right)$, and intensity of search $\left(I_{c S}\right)$, we show that C57BI/6J and $\mathrm{DBA} / 2 \mathrm{~J}$ mice have memory for both platform locations, whereas the residence time measure shows that on PD5, C57BI/6J mice do not search in old platform location above chance and that DBA/2J mice did not learn either of the platform location. (a) and (c) shows the divergence heat map for the population of $\mathrm{C} 57 \mathrm{BI} / 6 \mathrm{~J}$ and DBA/2J mice $(n=5)$. C57BI/6J mice show a distinct peak on PD3 and two peaks on PD5. In contrast, $\mathrm{DBA} / 2 \mathrm{~J}$ mice do not show a convergence peak on PD3 but show two peaks on PD5. We consider both peaks on PD5 for further analysis and label the peak in NE quadrant/new platform location as Peak\#1 and peak in SW quadrant/old platform location as Peak\#2. (b) and (d) are the error in divergence contributed by variation within the population. A Gaussian blur (radius $=4.75$ ) was applied for visualizing the sampled pixels. The part figure (e) describes the accuracy of search center for C57BI/6J on PD3 and PD5. C57BI/6J show a high degree of accuracy (solid black bar, $\alpha=86 \pm 2.8$ ) on PD3. On PD5, the accuracy of Peak\#1 measured with respect to the target platform (NE quadrant; solid red bar: $\alpha_{c s}=86 \%$ $\pm 1 \%$ ), as well as the accuracy of Peak\#2 measured with respect to the conflict platform location (SW quadrant; solid blue bar: $\alpha_{c s}=86 \% \pm 1 \%$ ) is close to maximum. These results indicate that Bl6 mice have a highly accurate and precise spatial memory for both the platform locations, that is, target location for PD5 and the conflict location on PD3. One-way ANOVA $F_{2,12}=0.0034, p>.05$. The part figure (f) describes the accuracy of search center for DBA/2J mice on PD3 and PD5. The absence of a convergence peak on PD3 indicates that DBA/2J do not possess spatial memory for the PD3 platform location. However, on PD5, DBA/2J mice have two search centers as shown in the convergence heat map. The accuracy of Peak\#1 measured with respect to the target platform (NE quadrant; Solid red bar: $\alpha=90 \% \pm 3 \%$ ), as well as the accuracy of the Peak\#2 measured with respect to the conflict platform location (SW quadrant; solid blue bar: $\alpha=86 \% \pm 3 \%$ ) is maximum. One-way ANOVA $F_{1,8}=1.09, p>.05$. Thus, illustrating the ability of our method to detect and describe the aspect of spatial memory corresponding to two distinct platform locations. Dashed line shows the chance accuracy value (64\%) in plots (e) and (f). Two-way ANOVA done for comparing the accuracy on PD5 with strain and peaks (platform locations) as factors showed that the means are not significantly different and there is no significant interaction (strain $F_{1,16}=0.78, p>.05$, peak: $F_{1,16}=0.897, p>.05$, strain $\times$ peak interaction: $F_{1,16}=0.973, p>.05$ (see Table S14b). The part figure (g) describes the uncertainty in search for C57BI/6J. On PD3, the relative search diameter is (diagonal black bar, $\delta_{R S}=4.43 \pm 0.15$ ). The relative search diameters of Peak\#1 new PL (diagonal red bar, $\delta_{R S}=3.45 \pm 0.048$ ) and Peak\#2 old PL (diagonal blue bar, $\delta_{R S}=2.75 \pm 0.039$ ) are not statistically different (one-way ANOVA $F_{2,12}=85, p<.001$ ), thus, the uncertainty in search is similar for both the platform locations (SW and $\mathrm{NE}$ ). The part figure $(\mathrm{h})$ describes the uncertainty in search for DBA/2J mice. The relative search diameters of Peak\#1 new PL (diagonal red bar, $\delta_{R S}=4.66 \pm 0.16$ ) and Peak\#2 old PL (diagonal blue bar, $\delta_{R S}=4.27 \pm 0.15$ ) are not statistically different (one-way ANOVA $F_{1,8}=3.18, p>.05$ ), thus, the uncertainty in search is similar for both the platform locations (SW and NE). Two-way ANOVA for mean uncertainty on PD5 with strain and peak as two factors showed that the uncertainty is different across the strain $\left(F_{1,16}=148, p<.001\right)$, and for location of the peaks $\left(F_{1,16}=23\right.$, $p<.001)$ with no significant interaction $\left(F_{1,16}=1.993, p>.05\right)$. From post hoc analysis we see the search diameter is lesser for the older location then the new one (see Table S15b) reduces the uncertainty in search area. (i) Relative intensity of search shows C57BI/6J mice maximally search at platform location on PD3 (hatched black bar, 100\% $\pm 3 \%$ ) while on PD5 they search at both the new platform location (hatched red bar, $100 \%$ $\pm 1 \%$ ) as well as the old location (hatched blue bar, $12.7 \% \pm 0.2 \%$ ). However, the intensity of search is higher for new location (one-way ANOVA $\left.F_{2,12}=592, p<.001\right)$. (j) Relative intensity of search of DBA/2J mice on PD5. The relative intensity of search for the platform at the target location (NE; hatched red bar, $66 \% \pm 2 \%$ ) is significantly more than at the conflict location (SW; hatched blue bar, $49 \% \pm 2 \%$; one-way ANOVA $\left.F_{1,8}=38, p<.001\right)$. Thus, the mice search more intently in the target location, compared with the conflict location as a result of goal reversal training. We note that intensity of search for the old platform location in DBA/2J is considerably more compared with the C57BI/6J. Two-way ANOVA for relative search intensity with strain and peak as two factors show that difference in means across the strains is not significant: $\left(F_{1,16}=0.17, p>.05\right)$ but mean intensity of search for the peaks are significantly different $\left(F_{1,16}=1127, p<.001\right)$ with a significant interaction $\left(F_{1,16}=510, p<.001\right.$ ) between the factors. Post hoc analysis (see Table S16b) shows that goal reversal is complete for C57BI/6J as indicated by lower search intensity for the old platform location while DBA/2J mice shows significantly more freezing at the old platform location. We estimate the efficiency of goal reversal training as $\left(I_{\text {new }}-I_{\text {old }}\right) /\left(I_{\text {new }}+I_{\text {old }}\right)$. Thus, a value of 1 would indicate complete goal reversal while -1 would indicate no reversal with zero indicating that there is a conflict. Here we find this efficiency for $\mathrm{C} 57 \mathrm{BI} / 6 \mathrm{~J}$ to be $\sim 0.78$ and DBA/2J to $\sim 0.15$. $(\mathrm{k})$ and $(\mathrm{m})$ The zone and quadrant residence time measure on PD5 for C57BL/6J mice. Both measures show that the mice spend significantly more time in the target location (see Figure S4 and the corresponding tables), whereas it resides in the conflict location at chance level. Thus, the residence time-based measures are unable to detect the presence of spatial memory for the older platform location. (I) and ( $\mathrm{n}$ ) The zone (solid bar) and quadrant (dotted bar) residence time measure PD5 for DBA/2J mice. DBA/2J mice occupy the target zone or quadrant at chance level. Thus, the measures are unable to detect the presence of spatial memory for both platform training locations. Dashed line represents the chance value $(1.11 \%$ and $25 \%$, respectively) in plots $(k-n)$ 
search area on PD5 is comparable to that of their WT littermates on PD3. We also show that the Ptpn $11^{\mathrm{N} 308 \mathrm{D} /+}$ mouse model of NS have a more subtle spatial learning and memory deficit in the MWM that was not easily detected by conventional measures. Additionally, treatment with a MEK inhibitor rescued the learning and memory deficits in Ptpn $11^{\mathrm{N} 308 \mathrm{D} /+}$ mice, adding to the evidence suggesting that inhibitors of this kinase could be used to treat cognitive deficits in patients. Lastly, we investigated whether the divergence field property on the velocity-based measure could detect the presence of multiple search centers, reflecting multiple spatial memories, in a MWM goal reversal data set. The two strains of mice (C57BI/6J and DBA/2J) trained in this goal reversal task revealed the presence of two search centers, corresponding to the old as well as the new target platform locations. Quantification of the difference in strength or nature of these two spatial memories, in terms of the extent of intention of search at the two locations or search centers, showed that both strains of mice prefer to search at the most recently trained platform location, and that acquisition of the new memory for the platform location did not disrupt memory for the first location.

To illustrate the sensitivity and advantage of metrics introduced here, we used multiple MWM data sets. We show that our methods can detect subtle memory differences previously undetected using occupancy-based quadrant, platform, proximity, or entropy measures. For comparison we have provided some of the commonly used measures for the same data sets as Supporting Information. Figure S4 compiles the residence time while Figure $\mathrm{S} 5$ shows the proximity and entropy. We present the results from the statistical comparisons as table in Supporting Information. We compared the estimates of SNR of these measures using Ptpn $11^{\mathrm{N} 308 \mathrm{D} /+}$ dataset in Figure S7.

Further, we note that the three metrics we introduced here are independent of each other and are sufficient to quantify spatial learning and memory. To illustrate this, we consider a real-life navigational task of visiting a favorite café located at block " $A$ " between intersections "l" and "II." In this context if the person remembers and goes to block " $\mathrm{B}$ " then accuracy captures the difference between the true location of the café and the actual location person went to. On reaching the place and not finding the cafe the person back tracks and searches around the place. The extent of area within which the person searches reflect their own estimate of how erroneous their memory for the location is. In this example the person knows the café is between two intersections but not remembering the block. Uncertainty captures this effect in our measure. Finally, the intensity of the search at block "B" captures the probability and hence the intention of that the person to target the search for the café at that point.

Moreover, we argue that the metrics introduced here reflect more closely spatial learning and memory than previous measures. For example, our methods reveal whether rodents use single or multiple search strategies in a trial, as well as the combined effect of the search results in swimming toward a particular region or location. This effect of swimming toward a given location is detected as a convergence peak or hotspot (i.e., the divergence field property is not preferentially sensitive or dependent on a particular search strategy). Additionally, since divergence measures the change in velocity in space, differences in absolute speed between strains of mice is not a major confound in our spatial learning and memory measures, as much as they confound the interpretation of previous measures (i.e., the divergence field property can dissociate a memory deficit from a motor deficit). Differences in swim speed affect many of the conventional measures since speed affects quadrant occupancy and area covered during searches. We note that moderate differences in swim speed do not necessarily affect training since the mice are usually left in the pool until they reach the platform. Both fast as well as slow swimmers can reach the platform during training. On learning the platform location, the mice normally reach the platform in one-tenth of the maximum training duration (usually $60 \mathrm{~s}$ ). Thus, with our measures, only mice that are considerably slower (>10 times slower) fail to reach the platform during this time, and thus only in this rare case would slow motor performance affect our spatial learning and memory measures. On the other hand, conventional measures such as quadrant occupancy, platform crossings and proximity, are dependent on total occupancy, and therefore are affected by changes in swim speed. However, the divergence of vector field measure introduced here reflects how the vectors are changing in space rather than the magnitude of the vectors per se. This minimizes, if not eliminates, the effects of difference in swim speeds. Thus, the proposed metrics, based on divergence of vector fields, improve the sensitivity of measuring memory performance by uncoupling the confounding effects of non-memory related processes, such as swimming speed, from measures of spatial learning and memory.

While we have presented the description, derivation, and potential utility of both the divergence and curl vector field properties, here we focused our analyses on developing and highlighting the power of the metrics derived from divergence vector field properties. In this study we have used the water maze as a tool in a traditional version of reference memory task. However, the water maze has been utilized/adapted to study a wide variety of other tasks such as delayed place to match task, everyday memory, and so on (Rossato et al., 2018; Steele \& Morris, 1999). We believe our metrics can be utilized directly or adapted appropriately to assess spatial memory in these tasks, but this remains to be tested.

All together our studies demonstrate that the measures introduced here are capable of extracting new information from readily accessible analyses of performance in the MWM, and consequently that they are capable of yielding novel insights that would be otherwise overlook by older measures of this widely used task for spatial learning and memory.

\section{5 | MATERIALS AND METHODS}

\subsection{Animal information}

BALB/cJ (Stock no: 000651) and SWR/J (SWR/J Stock no: 000689) inbred mice were obtained from Jackson Laboratory, The United States and maintained at the Central Animal Facility, IISc. All protocols were approved by the Institute's Animal Ethics Committee. 
For the experiments using NS mice, we used both transgenic and viral-induced mice models. NS virus-induced mice models were created by the induction of adeno-associated virus (AAV)-mediated expression of the NS-associated mutation (PTPN11 ${ }^{\text {D61G }}$ ) through bilateral injection into the dorsal CA regions of the hippocampus of 3-month-old $\mathrm{C} 57 \mathrm{BI} / 6 \mathrm{~J}$ male mice. To construct pAAV-hSynPTPN11 ${ }^{\text {D61G }}$-HA-T2A-tdTomato plasmid, PTPN11 ${ }^{\text {D61G-HA-T2A }}$ tdTomato was synthesized by using recombinant PCR and inserted into the AAV vector pAAV-hSyn-eGFP.WPRE.bGH vector (Addgene \#105539), replacing eGFP between Nhel and Hindlll site.

The NS mice were trained as reported previously (Lee et al., 2014). Briefly, training sessions comprised four trials (two blocks with two trials each) per day, with 1-min intertrial interval and 45-min interblock interval. Animals were allowed to search the platform for $60 \mathrm{~s}$ or until reaching the platform, as shown in the original article. The probe tests were done immediately after the completion of training on days 3, 5, 7, or 11, depending on the experiment. For the experiments with MEK inhibitor, SL327 (32 mg/kg, DMSO) was injected intraperitonially everyday $30 \mathrm{~min}$ before MWM training.

\section{2 | Generation of velocity-based vector field via surface fit}

We describe the swim trajectories in terms of the velocity component along the occupancy center for all pixels sampled by the mice. The value at each pixel is normalized to the number of times it has been sampled (i.e., the mean value at the pixel).

We perform a $5^{\text {th }}$ order polynomial surface fit (ImageJ Polynomial Surface Fit plugin) using an ROI of sampled pixels. The resultant surface is a continuous surface representing the velocity-based vector field. We require that the fit interpolates values for unsampled pixels within the sampled pixels ROI to compute the partial differential along the horizontal and vertical axis of the pool reference frame. Since the regions outside the ROI are not sampled, we do not consider it to be a part of the vector field. The imposed rectangular selection ROI during this fit results in cropped rectangular ROI. We modified and used the Differentials Plugin (Unser, 1999) in ImageJ to obtain the components of divergence. The general workflow is illustrated in Figure S3. A detailed step by step procedure is listed in Supporting Information Methods_1 along with usage instructions for the corresponding codes, plugins and macros. These details together with the source code is also available in our laboratory git hub page (https://github.com/ TheNeurodynamicsLab/SpatialMemory_VectorAnalysis_Code/wiki).

\section{3 | Estimation of error in generated velocity-based vector field}

The error in $5^{\text {th }}$ order polynomial surface fit is described by estimating the goodness of surface fit. The reduced Chi-sq $\left(\chi_{v}^{2}\right)$ describes the variance in the velocity-based measure among the population of mice as well as the variance in the estimate of fit values at each pixel.

$$
\chi_{v}^{2}=\frac{\chi^{2}}{v}=\frac{\sum_{i=1}^{n} \frac{\left(y_{i}-y\left(x_{i}\right)\right)^{2}}{\sigma_{i}^{2}}}{n-m}
$$

where $y_{i}$ is the actual value at the pixel $i, y\left(x_{i}\right)$ is the fit value at the pixel $i, \sigma_{i}{ }^{2}$ is the variance in velocity-based measure value at the pixel $i ; v$ is the degree of freedom defined as the difference between the number of points sampled on the surface $(n)$ minus the number of fitted parameters $(m)$. In case of a 5 th order polynomial equation, there are 14 coefficients or fitted parameters, hence the degree of freedom $v=n-14$.

\subsection{Estimation of error in field properties}

We represent the estimated error in the field properties as a percent error in residual of the surface fit.

$$
\text { Residualsumsq }=\mathrm{RSS}=\sum_{i=1}^{n}\left(y_{i}-y\left(x_{i}\right)\right)^{2}
$$

$$
\text { Weighted residualsumsq }=\text { wRSS }=\sum_{i=1}^{n} \frac{w_{i}\left(y_{i}-y\left(x_{i}\right)\right)^{2}}{\sum_{i=1}^{n} w_{i}}
$$

where $w_{i}=\frac{1}{\sigma_{i}^{2}}$

$$
\text { Percent error }=100 \sum_{i=1}^{n} \frac{w_{i}\left(y_{i}-y\left(x_{i}\right)\right)^{2}}{y_{i} \sum_{i=1}^{n} w_{i}}
$$

where $y_{i}$ is the actual value at the pixel $i, y\left(x_{i}\right)$ is the fit value at the pixel $i, \sigma_{i}^{2}$ is the variance in velocity-based measure value at the pixel $i$.

Hence, we calculate the total error at each pixel of the field property as the sum of the fractional errors contributed by the $x$ components and $y$-components with respect to the pool reference frame, given by,

$$
\sigma_{I}=\sqrt{\sigma_{x}^{2}+\sigma_{y}^{2}}
$$

We utilize the mode of the distribution of error values to best represent the most likely error estimate. We propagate this mode value of error when representing the derived parameters/metrics, that is, accuracy of search, uncertainty of search, and intensity of search.

\subsection{Identification of putative search centers}

We identify the putative search center as the global minima of the divergence heat map. The obtained divergence heat map is separated into two images, each containing either only positive values (divergence values) or negative values (convergence values). The image with negative values is inverted to convert the minima to maxima for visual representation. Next, we normalize the intensity value to the maximum intensity value. We locate the maxima using the ImageJ's plugin 
Maximum Finder class. The algorithm is set such that a maximum is identified if and only if the point has a peak value difference that is more than $0.1 \%$ and that the point is not an edge.

\section{6 | Estimation of FWHM of convergence peak}

We estimate the FWHM of a convergence peak as previously described (Waithe, n.d.; 2D Gaussian fitting macro [Fiji/lmageJ] for multiple signals, BIII). Briefly, the identified putative search center is fit to a linearized 2D-Gaussian function to obtain the FWHM in $x$-axis and $y$-axis. The size of the peak is initialized to 20 pixels before performing custom fit using ImageJ's Curve Fitter class implementing Simplex algorithm.

\subsection{Statistical analysis}

All statistical analysis was carried out in Origin (v2020b or 2021b). To perform two-way ANOVA on summarized statistics (i.e., ANOVA using mean and SEM), the raw data was recreated for " $n$ " individual mice by randomly sampling from a normal distribution using the population mean and SD of the metric being analyzed using rnorm() function of R-software. Based on ANOVA results, post hoc analysis was performed to carry out pair-wise comparisons. The data distribution was assumed to be normal. All data are represented as mean \pm SEM. We note that the polynomial surface fits as such can be compared for establishing the differences, however we took the resampling approach to parallel the conventional hypothesis testing and to compare the measures rather than the surfaces.

\section{ACKNOWLEDGMENTS}

The authors thank the funding source for their support and Prof Kempermann and Prof. Rupert Overall, CRTD, Dresden for sharing the C57BI/6J and DBA/2J data, Dr. Hyun-Hee Ryu for AAV vector construction. We also thank Prof. Chandra Murthy, IISc for reading through the manuscript and providing his valuable comments on the manuscript. We especially acknowledge his suggestion for estimating the field when there are multiple isolated areas of residence. We thank the CAF, IISc for providing and maintaining the mice for experiments performed at IISc.

\section{CONFLICT OF INTEREST}

The authors declare that they have no competing interests.

\section{AUTHOR CONTRIBUTIONS}

Meenakshi Prabod Kumar, Dhruv Mehrotra, N. Nruthyathi, and Balaji Jayaprakash designed the water maze experiment for BALB/cJ and SWR/J mice. Dhruv Mehrotra and N. Nruthyathi collected the water maze data for BALB/cJ and SWR/J mice. Yong-Seok Lee and Alcino Silva designed the SL327 rescue experiments with Ptpn11 N308D/+ mice and provided the Noonan mouse model data. Yong-Seok Lee and Daniel Almeida-Filho performed the Noonan mouse model experiments in Alcino Silva's lab. Meenakshi Prabod Kumar and Balaji Jayaprakash developed the theory, wrote the program for data extraction and analysis, analyzed the data, and authored the manuscript. Yong-Seok Lee, Daniel Almeida-Filho, and Alcino Silva read and provided corrections for the manuscript.

\section{ETHICS APPROVAL STATEMENT}

All protocols were approved by the Institute Animal Ethics Committee.

\section{DATA AVAILABILITY STATEMENT}

The datasets used and/or analyzed in the current study are available after publication from the corresponding author on written request. The software, workflow along with the codes developed are available in the github (https://github.com/neurodynamicslab).

\section{ORCID}

Prabod Kumar Meenakshi (D) https://orcid.org/0000-0002-0711-5762 Dhruv Mehrotra (D) https://orcid.org/0000-0001-5813-3218 J. Balaji DiD https://orcid.org/0000-0002-4442-6981

\section{REFERENCES}

Andersen, P., Morris, R., Amaral, D., Bliss, T., \& O'Keefe, J. (2009). The hippocampus book. Oxford University Press. https://doi.org/10.1093/ acprof:oso/9780195100273.001.0001

Araki, T., Chan, G., Newbigging, S., Morikawa, L., Bronson, R., \& Neel, B. G. (2009). Noonan syndrome cardiac defects are caused by PTPN11 acting in endocardium to enhance endocardial-mesenchymal transformation. Proceedings of the National Academy of Sciences of the United States of America, 106(12), 4736-4741. https://doi.org/10.1073/pnas.0810053106

Araki, T., Mohi, M. G., Ismat, F. A., Bronson, R. T., Williams, I. R., Kutok, J. L., Yang, W., Pao, L. I., Gilliland, D. G., Epstein, J. A., \& Neel, B. G. (2004). Mouse model of Noonan syndrome reveals cell type- and gene dosage-dependent effects of Ptpn11 mutation. Nature Medicine, 10(8), 849-857. https://doi.org/10.1038/nm1084

Brandeis, R., Brandys, Y., \& Yehuda, S. (1989). The use of the Morris water maze in the study of memory and learning. International Journal of Neuroscience, 48, 29-69. https://doi.org/10.3109/00207458909002151

Cooke, M. B., O'Leary, T. P., Harris, P., Brown, R. E., \& Snyder, J. S. (2019). Pathfinder: Open source software for analyzing spatial navigation search strategies. F1000Research, 8, 8. https://doi.org/10.12688/ f1000research.20352.1

Dalm, S., Grootendorst, J., de Kloet, E. R., \& Oitzl, M. S. (2000). Quantification of swim patterns in the Morris water maze. Behavior Research Methods, Instruments, and Computers, 32(1), 134-139. https://doi.org/ 10.3758/BF03200795

D'Hooge, R., \& De Deyn, P. P. (2001). Applications of the Morris water maze in the study of learning and memory. Brain Research Reviews, 36(1), 60-90. https://doi.org/10.1016/S0165-0173(01)00067-4

Eichenbaum, H., \& Cohen, N. J. (2014). Can we reconcile the declarative memory and spatial navigation views on hippocampal function? Neuron, 83, 764-770.

Eichenbaum, H. B. (2017). The role of the hippocampus in navigation is memory. Journal of Neurophysiology, 117(4), 1785-1796. https://doi. org/10.1152/JN.00005.2017

Gallagher, M., Burwell, R., \& Burchinal, M. R. (1993). Severity of spatial learning impairment in aging: Development of a learning index for performance in the Morris water maze. Behavioral Neuroscience, 107(4), 618-626. https://doi.org/10.1037//0735-7044.107.4.618

Garthe, A., Behr, J., \& Kempermann, G. (2009). Adult-generated hippocampal neurons allow the flexible use of spatially precise learning strategies. PLoS One, 4(5), 5464. https://doi.org/10.1371/journal.pone.0005464 
Gehring, T. V., Luksys, G., Sandi, C., \& Vasilaki, E. (2015). Detailed classification of swimming paths in the Morris water maze: Multiple strategies within one trial. Scientific Reports, 5(1), 14562. https://doi.org/10. 1038/srep14562

Graziano, A., Petrosini, L., \& Bartoletti, A. (2003). Automatic recognition of explorative strategies in the Morris water maze. Journal of Neuroscience Methods, 130(1), 33-44. https://doi.org/10.1016/S0165-0270(03)00187-0

Lee, Y. S., Ehninger, D., Zhou, M., Oh, J. Y., Kang, M., Kwak, C., Ryu, H. H., Butz, D., Araki, T., Cai, Y., Balaji, J., Sano, Y., Nam, I., Kim, H. K., Kaang, B. K., Burger, C., Neel, B. G., \& Silva, A. J. (2014). Mechanism and treatment for learning and memory deficits in mouse models of Noonan syndrome. Nature Neuroscience, 17(12), 1736-1743. https:// doi.org/10.1038/nn.3863

Maei, H. R., Zaslavsky, K., Teixeira, C. M., \& Frankland, P. W. (2009). What is the most sensitive measure of water maze probe test performance? Frontiers in Integrative Neuroscience, 3, 4. https://doi.org/10.3389/ neuro.07.004.2009

Maei, H. R., Zaslavsky, K., Wang, A. H., Yiu, A. P., Teixeira, C. M., Josselyn, S. A., \& Frankland, P. W. (2009). Development and validation of a sensitive entropy-based measure for the water maze. Frontiers in Integrative Neuroscience, 3, 33. https://doi.org/10.3389/neuro.07.033. 2009

Morris, R. (1984). Developments of a water-maze procedure for studying spatial learning in the rat. Journal of Neuroscience Methods, 11(1), 4760. https://doi.org/10.1016/0165-0270(84)90007-4

Morris, R. G. M. (1981). Spatial localization does not require the presence of local cues. Learning and Motivation, 12(2), 239-260. https://doi.org/ 10.1016/0023-9690(81)90020-5

Overall, R. W., Zocher, S., Garthe, A., \& Kempermann, G. (2020). Rtrack: A software package for reproducible automated water maze analysis. bioRxiv Preprints. https://doi.org/10.1101/2020.02.27.967372

Paylor, R., Tracy, R., Wehner, J., \& Rudy, J. W. (1994). DBA/2 and C57BL/6 mice differ in contextual fear but not auditory fear conditioning. Behavioral Neuroscience, 108(4), 810-817. https://doi.org/10. 1037/0735-7044.108.4.810

Pereira, I. T., \& Burwell, R. D. (2015). Using the spatial learning index to evaluate performance on the water maze. Behavioral Neuroscience, 129(4), 533-539. https://doi.org/10.1037/bne0000078

Petrosini, L., Leggio, M. G., \& Molinari, M. (1998). The cerebellum in the spatial problem solving: A co-star or a guest star? Progress in Neurobiology, 56(2), 191-210. https://doi.org/10.1016/S0301-0082(98)00036-7

Redish, A. D., \& Touretzky, D. S. (1998). The role of the hippocampus in solving the Morris water maze. Neural Computation, 10(1), 73-111. https://doi.org/10.1162/089976698300017908

Romano, A. A., Allanson, J. E., Dahlgren, J., Gelb, B. D., Hall, B., Pierpont, M. E., Roberts, A. E., Robinson, W., Takemoto, C. M., \& Noonan, J. A. (2010). Noonan syndrome: Clinical features, diagnosis, and management guidelines. Pediatrics, 126(4), 746-759. https://doi. org/10.1542/peds.2009-3207

Rossato, J. I., Moreno, A., Genzel, L., Yamasaki, M., Takeuchi, T., Canals, S., \& Morris, R. G. M. (2018). Silent learning. Current Biology, 28(21), 3508-3515. https://doi.org/10.1016/j.cub.2018.09.012

Sahoo, P. K., Soltani, S., \& Wong, A. K. C. (1988). A survey of thresholding techniques. Computer Vision, Graphics and Image Processing, 41(2), 233-260. https://doi.org/10.1016/0734-189X(88)90022-9

Steele, R. J., \& Morris, R. G. M. (1999). Delay-dependent impairment of a matching-to-place task with chronic and intrahippocampal infusion of the NMDA-antagonist D-AP5. Hippocampus, 9(2), 118-136. https:// doi.org/10.1002/(SICI)1098-1063(1999)9:2<118::AID-HIPO4>3.0. $\mathrm{CO} ; 2-8$

Tartaglia, M., \& Gelb, B. D. (2005). Noonan syndrome and related disorders: Genetics and pathogenesis. Annual Review of Genomics and Human Genetics, 6, 45-68. https://doi.org/10.1146/annurev.genom.6. 080604.162305
Unser, M. (1999). Splines: A perfect fit for signal and image processing. IEEE Signal Processing Magazine, 16(6), 22-38. https://doi.org/10. 1109/79.799930

Upchurch, M., \& Wehner, J. M. (1988). Differences between inbred strains of mice in Morris water maze performance. Behavior Genetics, 18(1), 55-68. https://doi.org/10.1007/BF01067075

Vouros, A., Gehring, T. V., Szydlowska, K., Janusz, A., Tu, Z., Croucher, M., Lukasiuk, K., Konopka, W., Sandi, C., \& Vasilaki, E. (2018). A generalised framework for detailed classification of swimming paths inside the Morris water maze. Scientific Reports, 8(1), 15089. https://doi.org/ 10.1038/s41598-018-33456-1

Waithe, D. (n.d.). 2D Gaussian fitting macro (Fiji/ImageJ) for multiple signals, BIII. https://biii.eu/2d-gaussian-fitting-macro-fijimagej-multiplesignals

Wolfer, D. P., \& Lipp, H. P. (1992). A new computer program for detailed off-line analysis of swimming navigation in the Morris water maze. Journal of Neuroscience Methods, 41(1), 65-74. https://doi.org/10. 1016/0165-0270(92)90124-V

\section{SUPPORTING INFORMATION}

Additional supporting information may be found in the online version of the article at the publisher's website.

How to cite this article: Meenakshi, P. K., Mehrotra, D., Nruthyathi, N., Almeida-Filho, D., Lee, Y.-S., Silva, A., \& Balaji, J. (2022). Novel measures of Morris water maze performance that use vector field maps to assess accuracy, uncertainty, and intention of navigational searches. Hippocampus, 1-22. https://doi.org/10.1002/hipo.23404

\section{APPENDIX A}

\section{A.1 | Theory}

A.1.1 | Velocity-based vector fields for describing the intentional movement of rodent swim trajectories

i. Description of velocity vector fields:

We first developed an analytical framework for describing the swim trajectories in terms of a velocity vector field. The position vector $\left(\overrightarrow{P_{t}}\right)$ represents the point at which the rodent is located at a given time (" $t$ "-usually measured in video frames). The origin of the reference frame $O(0,0)$ is set to be the top left corner (Figure 1a). Thus, from an image taken from the top, the pixel coordinates $\left(P_{x}, P_{y}\right)$ can be used to write $\left(P_{t}\right)$ as follows:

$$
\overrightarrow{P_{t}}=P_{x} \hat{i}+P_{y} \hat{j}
$$

where $P_{x}$ and $P_{y}$ are the distance in pixels from top left corner in the image.

For convenience, we define a displacement vector $\left(\overrightarrow{D_{t}}\right)$ that provides a relation of the rodent's position with respect to a point 
of interest, for example, the platform. This is calculated as the difference between the platform vector $\overrightarrow{P_{L}}$ and the instantaneous position vector $\left(\overrightarrow{P_{t}}\right)$ defined above (Figure $1 b$ ).

$$
\overrightarrow{D_{t}}=\overrightarrow{P_{L}}-\overrightarrow{P_{t}}
$$

The velocity vector $\left(\overrightarrow{V_{t}}=\frac{d}{d t} \overrightarrow{D_{t}}\right)$ is a metric that describes the movement of the rodent. Since we make these measurements from time lapse videos that are recorded at uniform frame rates, we can calculate the velocity vector as the difference between two consecutive displacement vectors (Figure 1c).

$$
\overrightarrow{V_{t}}=\overrightarrow{D_{t+1}}-\overrightarrow{D_{t}}
$$

ii. Definition of occupancy center:

Since we measure the component of velocity of the animal that is directed toward reaching the "goal," it is convenient to define the occupancy center $P_{o c}$ as the point where the likelihood of finding the animal is high given its trajectory. We calculate $P_{o c}$ as the center of mass obtained from the residence time map that is segmented using a maximum entropy threshold. Maximum entropy (ME) segmentation determines a threshold $(T)$ that has the maximum entropy of the background $\left(H_{B}\right)$ and foreground $\left(H_{F}\right)$ in an image (Sahoo et al., 1988).

$$
\begin{aligned}
& H_{B}=-\sum_{i=1}^{T} p_{i} \log _{2}\left(p_{i}\right) \\
& H_{F}=-\sum_{i=T+1}^{n} p_{i} \log _{2}\left(p_{i}\right)
\end{aligned}
$$

where $p_{i}$ is the number of pixels with value $i$ divided by the total number of pixels in their respective segments $\left(H_{B}\right.$ or $\left.H_{F}\right)$, and $n$ is the maximal possible intensity in the image. This enables us to segment out the regions with zero or low occupancy from regions of high occupancy without bias. The use of ME segmentation increases the fidelity and hence the confidence in our estimate of the occupancy center as the most likely resided region based on a rodent's swim trajectory. This is given by (Figure 1f).

$$
x_{c m}=\frac{\sum_{i=1}^{n} x_{i} l_{i}}{\sum_{i=1}^{n} l_{i}} \text { and } y_{c m}=\frac{\sum_{i=1}^{n} y_{i} l_{i}}{\sum_{i=1}^{n} l_{i}} \text { where } I_{i}>T
$$

such that for the choosen $T$, the sum $\left(H_{B}+H_{F}\right)$ is maximum.

iii. Description of vector fields with respect to the occupancy center:

Using the occupancy center, we computed two different measures to describe the rodent's movement. First, the component of velocity vector along the occupancy center, a spatial property that measures the contribution of the movement at that location toward the occupancy center. This component is the vector projection or the velocity vector $V(t)$ along the direction of the occupancy center $\left(\widehat{d_{P_{o c}}}\right)$, and is given by (Figure $1 \mathrm{~d}$ )

$$
\overrightarrow{V_{\| t}}=\left|\overrightarrow{V_{t}}\right| \cos \theta_{v} \widehat{d_{P_{O C}}}
$$

where $\left|\overrightarrow{V_{t}}\right|$ is the magnitude of the velocity vector and $\theta_{v}$ is the angle between the velocity vector $\overrightarrow{V_{t}}$ and a unit vector pointing toward the occupancy center $\left(\widehat{d_{P_{\text {oc }}}}\right)$ from the current position.

Second, the velocity vector component orthogonal to the occupancy center is a spatial property that measures at that position, the contribution of the rodent's movement to the circular motion about the occupancy center. It is given by (Figure 1e)

$$
V_{\perp t}=\left|\overrightarrow{V_{t}}\right| \sin \theta_{v} \widehat{d_{P_{o c}}}
$$

where $\left|\overrightarrow{V_{t}}\right|$ is the magnitude of the velocity vector and $\theta_{v}$ is the angle between velocity vector $\overrightarrow{V_{t}}$ and the unit vector pointing toward the occupancy center $\left(\widehat{d_{P_{o c}}}\right)$. We note if ME segmentation yields multiple disconnected regions of comparable sizes then we need to consider multiple occupancy centers. In such cases we evaluate the velocity vector components toward each of them and take the weighted sum of their magnitudes. The weights are determined by the fractional area of the patch. In the current study we did not use such as approach.

Next, we resolved these vectors Equations (A4) and (A5) in a frame of reference defined with respect to the image of the pool. The origin is set at the top left corner of the image with right and down being the positive directions of its axis. Thus, the above vectors could be written as

$$
\begin{gathered}
V_{\| t}=V_{\| t} \cos \theta_{d} \widehat{i}+V_{\| t} \sin \theta_{d \dot{j}} \\
V_{\perp t}=V_{\perp t} \cos \theta_{d} \widehat{i}+V_{\perp t} \sin \theta_{d} \widehat{j}
\end{gathered}
$$

where $\theta_{d}$ is the angle, $\widehat{i}$ and $\widehat{j}$ are the unit vectors along the height and width of the image frame.

\section{A.2 Analytical expression of the putative search centers} obtained from divergence in velocity-based vector fields

i. Vector field properties:

Divergence of a vector field indicates the difference or change in flux around an infinitesimal volume. We reason that given a vector field $V_{\| t}$, the point of convergence ("sink" in a vector field) could represent the point where the animal intends to reach. We hypothesize that if a rodent intents to reach a specific location $P_{L}^{\prime}$ in the pool, then the velocity field would point inward from the 
region with progressively decreasing magnitude (as described in the introduction) around the point $P_{L}^{\prime}$ leading to a negative divergence value (i.e., indicating convergences) at $P_{L}^{\prime}$. However, in general $P_{L}^{\prime}$ need not necessarily coincide with the platform location or occupancy center, for they represent the center of a region where the rodent is most likely found, and not the actual platform location. We construct the vector field from vector projections (Equation (A1)) to measure and represent the intention of the animal to move toward the occupancy center as a function of its position in the pool. The heat map of the global minima of such a divergence would be indicative of the platform location as perceived by the animal. We calculate the divergence on the vector field describing the velocity along the occupancy center in the image reference frame as

$$
I_{\text {div }}(x, y)=\nabla \cdot V_{\| t}=\frac{\partial}{\partial x} V_{\| t} \cos \theta_{d}+\frac{\partial}{\partial y} V_{\| t} \sin \theta_{d}
$$

Similarly, we reason that the curl of a vector field could indicate the tendency of rotation of an object placed at that location, and hence we propose that it could be used to extract the tendency of the animal to circle around any point in the pool. For completion, we describe the curl, but in subsequent analyses, we restricted our analyses to divergence.

Curl of a field is formally defined as the circulation density at each point of the field (i.e., the extent of rotation about a point). Thus, curl on $V_{\perp t}$ vector field would be informative of the extent of circular movement that the animal makes about the perceived platform location while searching for it. Using our notation, the curl can be written as:

$$
I_{\text {curl }}(x, y)=\nabla \times V_{\perp t}=\left(\frac{\partial}{\partial x} V_{\perp t} \sin \theta_{d}-\frac{\partial}{\partial y} V_{\perp t} \cos \theta_{d}\right) \widehat{k}
$$

ii. Description of the putative search center $\left(P_{c s}\right)$ :

Search centers can be obtained from the divergence calculated on the vector field of the velocity component pointing toward the occupancy center. The convergence peaks in such a map are indicative of the search centers $\left(\overrightarrow{P_{c S}}\right)$ or the platform locations as perceived by the rodent.

We obtain the search center or the convergence point by estimating the minima of the divergence on the velocity-based measure. For a two-dimensional surface, we obtain the critical point $\left(x_{o}, y_{o}\right)$ by equating the first derivative to be zero and forcing the second derivative to be positive as follows:

$$
\overrightarrow{P_{c s}}=\sqrt{\left(x_{0}^{2}+y_{0}^{2}\right)} \text { for }
$$

(i) $\nabla I_{\text {div }}\left(x_{o}, y_{o}\right)=0$

$$
\text { (ii) } H=I_{d i v_{x x}}\left(x_{0}, y_{0}\right) I_{d i v_{y y}}\left(x_{0}, y_{0}\right)+I_{d i v_{x y}}\left(x_{0}, y_{0}\right)^{2}
$$

where $H>0$ and $I_{\operatorname{div}_{x x}}\left(x_{0}, y_{0}\right)>0$.

\section{A.3 | Metrics to assess spatial memory}

We define and use three measures that are independent of each other to assess spatial memory in terms of various attributes of the search center $\left(\overrightarrow{P_{c s}}\right)$. The three independent measures namely, including accuracy of search, uncertainty about the search center, and intensity at the search center, are described next.

i. Accuracy of the search center $\left(\alpha_{c s}\right)$ (Figure 1g):

Since the search center is indicative of the animal's perceived platform location, the distance between the search center and the platform location measures inaccuracy or error in the animal's ability to learn and remember the platform location. The difference in location measured in units of length can be difficult to compare across different laboratories, since the effective pool and platform sizes can vary between laboratories. To address this problem, we developed a percentage measure that can be used for comparisons of animals' performances across laboratories. First, we realized that the maximum error in accuracy that the animal can make can be captured by the distance between the platform center and the farthest pool boundary (e). Next, we defined the accuracy of search $\left(\alpha_{c s}\right)$ as 1 minus the fractional error, with fractional error being the ratio between the distance of the search center from the platform and the maximum error. Thus, the accuracy of the search center is given by

$$
\alpha_{c s}=100 \times \frac{\left(e-d_{c s}\right)}{e}
$$

where $d_{c s}=\left|\overrightarrow{P_{l}}-\overrightarrow{P_{c s}}\right|$.

Furthermore, to estimate the chance factor we consider the case of a naïve animal that does not have any knowledge of the platform location (e.g., an animal on its first day of training). At this point of training, animals usually exhibit wall-hugging behavior (i.e., they swim in circles close to the wall). In such a scenario, the animal is moving about the pool center as its navigational center, and by virtue of symmetry the pool center would reflect the search center. We make use of this fact to define the chance accuracy as the accuracy when the search center coincides with the pool center and is given by

$$
\alpha_{\text {chance }_{\text {p }}}=100 \times \frac{r}{e},
$$

where $r$ is the radius of the pool.

Thus, an accuracy value that is comparable to the chance factor (Equation (A10)) would reflect whether the animal is still exhibiting symmetric navigational behavior. As the rodent learns the rules of the water maze, this symmetry is broken. Comparisons of accuracy with the chance factor above yield a categorical measure of learning and memory in the maze. 
ii. Uncertainty about the search center $\left(\sigma_{c s}\right)$ (Figure $1 \mathrm{~h}$ ):

Apart from the location of the search center, the spread of the divergence values around the peak provide additional insights into the level of uncertainty in the internal representation of the platform position in the animal's performance. We calculated this by measuring Uncertainty about the search center and defined it as the FWHM of the convergence peak representing the search center. We assume that the convergence peak is a 2D Gaussian, and compute the FWHM by linearizing the 2D Gaussian equation as previously described (Waithe, n.d.; 2D Gaussian fitting macro [Fiji/ImageJ] for multiple signals, BIII). We express the uncertainty about the search center in terms of the relative search diameter, defined as a ratio of the major axis of the Gaussian ellipse to the platform diameter.

$$
d_{R S}=\frac{\sigma_{c S}}{d_{P_{L}}}
$$

iii. Intensity of search $\left(I_{c s}\right)$ (Figure 1 i):

The absolute search intensity $\left(a l_{c s}\right)$, defined as the magnitude of convergence at the search center, is a measure of the animal's searches in the search center, that is,

$$
a I_{c s}=-I_{\text {div }}\left(x_{0}, y_{0}\right)
$$

Additionally, we define the relative search intensity $\left(r l_{c s}\right)$ as the magnitude of convergence at the search center normalized to the maximum convergence seen anywhere in the pool. We propose that this measure reflects the fractional effort of the animal's search at the search center.

$$
r l_{c s}=\frac{I_{c s}}{I_{\max }}
$$

$I_{\text {div }}\left(x_{0}, y_{0}\right)$ and $I_{\max }$ are the maximum divergence inside the pool. 